\title{
Integral inequalities via Raina's fractional integrals operator with respect to a monotone function
}

\author{
Shu-Bo Chen', Saima Rashid², Zakia Hammouch³, Muhammad Aslam Noor ${ }^{4}$, Rehana Ashraf ${ }^{5}$ and \\ Yu-Ming Chu ${ }^{6,7^{*}}$
}

"Correspondence:

chuyuming2005@126.com

${ }^{6}$ Department of Mathematics,

Huzhou University, Huzhou, China

${ }^{7}$ Hunan Provincial Key Laboratory of

Mathematical Modeling and

Analysis in Engineering, Changsha

University of Science \& Technology,

Changsha, China

Full list of author information is

available at the end of the article

\section{Springer}

\begin{abstract}
We establish certain new fractional integral inequalities involving the Raina function for monotonicity of functions that are used with some traditional and forthright inequalities. Taking into consideration the generalized fractional integral with respect to a monotone function, we derive the Grüss and certain other associated variants by using well-known integral inequalities such as Young, Lah-Ribarič, and Jensen integral inequalities. In the concluding section, we present several special cases of fractional integral inequalities involving generalized Riemann-Liouville, $k$-fractional, Hadamard fractional, Katugampola fractional, (k,s)-fractional, and Riemann-Liouville-type fractional integral operators. Moreover, we also propose their pertinence with other related known outcomes.
\end{abstract}

MSC: 26D15;26D10

Keywords: Grüss inequality; Young inequality; Generalized fractional integral operator; Raina function

\section{Introduction and preliminaries}

The fractional calculus has gained importance during recent years because of its applications in science and engineering. Fractional-order differential equations are widely used in the model problems of nanoscale flow and heat transfer, diffusion, polymer physics, chemical physics, biophysics, medical sciences, turbulence, electric networks, electrochemistry of corrosion, and fluid flow through porous media [1-5]. Fractional integral inequalities associating functions of two or more independent variables play a crucial role in the continuous growth of the theory, methods, and applications of differential and integral equations. In view of wider applications, integral inequalities have received considerable attention. Recently, several refinements of fractional integral inequalities have been proposed, which are helpful in the study of distinct classes of differential and integral equations. These variants act as ready tools to investigate the classes of differential and integral equations [6-9].

(c) The Author(s) 2020. This article is licensed under a Creative Commons Attribution 4.0 International License, which permits use sharing, adaptation, distribution and reproduction in any medium or format, as long as you give appropriate credit to the original author(s) and the source, provide a link to the Creative Commons licence, and indicate if changes were made. The images or other third party material in this article are included in the article's Creative Commons licence, unless indicated otherwise in a credit line to the material. If material is not included in the article's Creative Commons licence and your intended use is not permitted by statutory regulation or exceeds the permitted use, you will need to obtain permission directly from the copyright holder. To view a copy of this licence, visit http://creativecommons.org/licenses/by/4.0/ 
It is well known that the Grüss-type inequalities in both continuous and discrete cases play a significant role in investigating the qualitative conduct of differential and difference equations, respectively, as well as several other fields of pure and applied analysis.

Getting this tendency, we present a novel version for the most aesthetic and useful Grüss-type inequality [10] and some other associated inequalities with respect to another function $\vartheta$ that could be progressively viable and, moreover, more appropriate than the previous ones. The Grüss inequality can be stated as follows.

Theorem 1.1 ([10]) Let $\phi_{1}, \phi_{2}, \psi_{1}, \psi_{2} \in \mathbb{R}$ with $\phi_{1}<\phi_{2}$ and $\psi_{1}<\psi_{2}$, and let $\mathcal{Q}_{1}, \mathcal{Q}_{2}$ : $\left[v_{1}, v_{2}\right] \rightarrow \mathbb{R}$ be two integrable functions such that $\phi_{1}<\mathcal{Q}_{1}(z)<\phi_{2}$ and $\psi_{1}<\mathcal{Q}_{2}(z)<\psi_{2}$ for all $z \in\left[v_{1}, v_{2}\right]$. Then we have the inequality

$$
\begin{aligned}
& \left|\frac{1}{v_{2}-v_{1}} \int_{v_{1}}^{v_{2}} \mathcal{Q}_{1}(z) \mathcal{Q}_{2}(z) d z-\frac{1}{\left(v_{2}-v_{1}\right)^{2}} \int_{v_{1}}^{v_{2}} \mathcal{Q}_{1}(z) d z \int_{v_{1}}^{v_{2}} \mathcal{Q}_{2}(z) d z\right| \\
& \quad \leq \frac{1}{4}\left(\phi_{2}-\phi_{1}\right)\left(\psi_{2}-\psi_{1}\right)
\end{aligned}
$$

with the best possible constant $1 / 4$.

Inequality (1.1) is a tremendous mechanism for investigating numerous scientific areas of research comprising engineering, fluid dynamics, biosciences, chaos, meteorology, vibration analysis, biochemistry, aerodynamics, and many more. There was a constant development of enthusiasm for such an area of research so as to address the issues of different utilizations of these variants [11-15]. The conventional theory of inequality is unable to clarify the true behavior of (1.1). A review of basic concepts of fractional integral inequalities and an understanding about the Grüss was presented by Dahmani et al. [16]. Rashid et al. $[17,18]$ formulated the governing inequality by using generalized $k$-fractional integral and generalized proportional fractional integral. Based on a monotone function, Rashid et al. [19] derived fractional integral inequalities by means of the generalized proportional fractional integral operator in the sense of another function. Very recently, Butt et al. [20] proposed novel fractional refinements of Čebyšev-Pólya-Szegö-type inequalities by using the Raina function in the kernel.

Now we evoke some preliminaries ideas, which help the readers in clear understanding.

Definition 1.2 Let $1<\mathfrak{p}<\infty$ and $\mathfrak{r}>0$. Then the mapping $\mathcal{Q}_{1}(t)$ is said to be in $L_{\mathfrak{p}, \mathfrak{r}}\left[v_{1}, v_{2}\right]$ if

$$
\left(\int_{v_{1}}^{v_{2}}\left|\mathcal{Q}_{1}(t)\right|^{\mathfrak{p}} t^{\mathfrak{r}} d t\right)^{1 / \mathfrak{p}}<\infty
$$

Definition 1.3 ([21]) Let $k>0$. Then the generalized gamma function $\Gamma_{k}$ is defined by

$$
\Gamma_{k}(z)=\lim _{n \rightarrow \infty} \frac{n ! k^{n}(n k)^{\frac{z}{k}-1}}{(z)_{n, k}} .
$$

The Mellin transform of the exponential function $e^{-\frac{t^{k}}{k}}$ is the $k$-gamma function given by $\Gamma_{k}(\alpha)=\int_{0}^{\infty} e^{-\frac{t^{k}}{k}} t^{\alpha-1} d t$. Also, $\Gamma_{k}(z+k)=z \Gamma_{k}(z), \Gamma(z)=\lim _{k \rightarrow 1} \Gamma_{k}(z)$, and $\Gamma_{k}(z)=k^{\frac{z}{k}-1} \Gamma\left(\frac{z}{k}\right)$. 
Definition 1.4 ([22]) The function $\mathcal{F}_{\rho, \lambda}^{\sigma, k}$ is defined by

$$
\begin{aligned}
\mathcal{F}_{\rho, \lambda}^{\sigma, k}(z) & =\mathcal{F}_{\rho, \lambda}^{(\sigma(0), \sigma(1), \ldots), k}(z) \\
& =\sum_{m^{\prime}=0}^{\infty} \frac{\sigma\left(m^{\prime}\right)}{k \Gamma_{k}\left(\rho k m^{\prime}+\lambda\right)} z^{m^{\prime}} \quad(\rho, \lambda>0, z \in \mathbb{C},|z|<\mathbb{R}),
\end{aligned}
$$

where $\mathbb{R}$ is a real positive constant, and $\sigma=\left(\sigma(1), \ldots, \sigma\left(m^{\prime}\right), \ldots\right)$ is a bounded sequence of positive real numbers.

Definition 1.5 ([23]) Let $k>0, \lambda>0, \rho>0$, and $\omega \in \mathbb{R}$, and let $\vartheta:\left[v_{1}, v_{2}\right] \rightarrow(0, \infty)$ be an increasing function such that $\vartheta^{\prime}$ is continuous on $\left(v_{1}, v_{2}\right)$. Then the left and right generalized $k$-fractional integrals of the function $\mathcal{Q}_{1}$ with respect to $\vartheta$ on $\left[v_{1}, v_{2}\right]$ are defined by

$$
\mathcal{J}_{\rho, \lambda, v_{1}^{+} ; \omega}^{\sigma, k, \vartheta} \mathcal{Q}_{1}(z)=\int_{v_{1}}^{z} \frac{\vartheta^{\prime}(t)}{(\vartheta(z)-\vartheta(t))^{1-\frac{\lambda}{k}}} \mathcal{F}_{\rho, \lambda}^{\sigma, k}\left[\omega(\vartheta(z)-\vartheta(t))^{\rho}\right] \mathcal{Q}_{1}(t) d t \quad\left(z>v_{1}\right)
$$

and

$$
\mathcal{J}_{\rho, \lambda, v_{2}^{+}, \omega}^{\sigma, k, \vartheta} \mathcal{Q}_{1}(z)=\int_{z}^{v_{2}} \frac{\vartheta^{\prime}(t)}{(\vartheta(t)-\vartheta(z))^{1-\frac{\lambda}{k}}} \mathcal{F}_{\rho, \lambda}^{\sigma, k}\left[\omega(\vartheta(t)-\vartheta(z))^{\rho}\right] \mathcal{Q}_{1}(t) d t \quad\left(z<v_{2}\right)
$$

respectively.

Remark 1.6 (see [23]) Some noteworthy particular cases of (1.4) and (1.5) are given as follows.

(a) If $k=1$, then operator (1.4) reduces to the generalized fractional integral

$$
\mathcal{J}_{\rho, \lambda, v_{1}^{+} ; \omega}^{\sigma, \mathcal{Q}_{1}}(z)=\int_{v_{1}}^{z} \frac{\vartheta^{\prime}(t)}{(\vartheta(z)-\vartheta(t))^{1-\lambda}} \mathcal{F}_{\rho, \lambda}^{\sigma}\left[\omega(\vartheta(z)-\vartheta(t))^{\rho}\right] \mathcal{Q}_{1}(t) d t \quad\left(z>v_{1}\right)
$$

(b) Let $\vartheta(t)=t$. Then operator (1.4) becomes the generalized $k$-fractional integral

$$
\mathcal{J}_{\rho, \lambda, v_{1}^{+} ; \omega}^{\sigma, k} \mathcal{Q}_{1}(z)=\int_{v_{1}}^{z}(z-t)^{1-\frac{\lambda}{k}} \mathcal{F}_{\rho, \lambda}^{\sigma, k}\left[\omega(z-t)^{\rho}\right] \mathcal{Q}_{1}(t) d t \quad\left(z>v_{1}\right)
$$

(c) If $\vartheta(t)=\ln t$, then operator (1.4) reduces to the Hadamard $k$-fractional integral

$$
\mathcal{J}_{\rho, \lambda, v_{1}^{+} ; \omega}^{\sigma, k} \mathcal{Q}_{1}(z)=\int_{v_{1}}^{z}\left(\ln \frac{z}{t}\right)^{1-\frac{\lambda}{k}} \mathcal{F}_{\rho, \lambda}^{\sigma, k}\left[\omega\left(\ln \frac{z}{t}\right)^{\rho}\right] \frac{\mathcal{Q}_{1}(t)}{t} d t \quad\left(z>v_{1}\right) .
$$

(d) Let $\vartheta(t)=\frac{t^{s+1}}{s+1}(s \in \mathbb{R} \backslash\{-1\})$. Then operator (1.4) becomes the generalized $(k, s)$ fractional integral

$$
s \mathcal{J}_{\rho, \lambda, v_{1}^{+} ; \omega}^{\sigma, k} \mathcal{Q}_{1}(z)=\int_{v_{1}}^{z} \frac{t^{s}\left(x^{s+1}-t^{s+1}\right)^{1-\frac{\lambda}{k}}}{(1+s)^{\frac{\lambda}{k}-1}} \mathcal{F}_{\rho, \lambda}^{\sigma, k}\left[\omega\left(\frac{z^{s+1}-t^{s+1}}{s+1}\right)^{\rho}\right] \mathcal{Q}_{1}(t) d t \quad\left(z>v_{1}\right)
$$


(e) Let $\vartheta(t)=t$ and $k=1$. Then operator (1.4) reduces to

$$
\mathcal{J}_{\rho, \lambda, v_{1}^{+} ; \omega}^{\sigma} \mathcal{Q}_{1}(z)=\int_{v_{1}}^{z}(z-t)^{1-\lambda} \mathcal{F}_{\rho, \lambda}^{\sigma}\left[\omega(z-t)^{\rho}\right] \mathcal{Q}_{1}(t) d t \quad\left(z>v_{1}\right)
$$

which was proposed by Raina et al. [22] and Agarwal [24].

Remark 1.7 Let $\omega=0, \lambda=\alpha$, and $\sigma(0)=1$ in Definition 1.5. Then we have the following particular cases:

(1) Taking $k=1$, we get the fractional integrals of [25];

(2) Taking $\vartheta(t)=t$, we obtain the $k$-fractional integrals of [26];

(3) Taking $\vartheta(t)=\ln t$ and $k=1$, we get the Hadamard fractional integrals of [25];

(4) Taking $\vartheta(t)=\frac{t^{s+1}}{s+1}(s \in \mathbb{R} \backslash\{-1\})$, we obtain the $(k, s)$-fractional integrals of [27];

(5) Taking $\vartheta(t)=\frac{t^{s+1}}{s+1}(s \in \mathbb{R} \backslash\{-1\})$ and $k=1$, we get the Katugampola fractional integrals of [28].

The principal purpose of this paper is deriving novel identities, integral inequalities including a Grüss-type inequality, and numerous other associated inequalities via generalized fractional integral inequalities with respect to other function $\vartheta$ by using Young's, weighted arithmetic and geometric mean inequalities, and so on. It is interesting that many particular cases can be revealed by using Remarks 1.6 and 1.7. Therefore it is necessary to propose the investigation of the generalized fractional integrals.

\section{Fractional Grüss-type inequalities}

To demonstrate the main consequences of this paper, we begin with certain integral inequalities and equalities for positive integrable functions with the generalized fractional integral operator having the well-known Raina function in its kernel.

Throughout this investigation, we use the following suppositions:

(i) $\vartheta:[0, \infty) \rightarrow(0, \infty)$ is an increasing function with continuous derivative $\vartheta^{\prime}$ on the interval $(0, \infty)$.

(ii) $\mathcal{A}_{\lambda}$ and $\mathcal{A}_{\delta}$ are defined by

$$
\mathcal{A}_{\lambda}(z)=(\vartheta(z))^{\frac{\lambda}{k}} \mathcal{F}_{\rho, \lambda+1}^{\sigma, k}\left(\omega(\vartheta(z))^{\rho}\right)
$$

and

$$
\mathcal{A}_{\delta}(z)=(\vartheta(z))^{\frac{\delta}{k}} \mathcal{F}_{\rho, \delta+1}^{\sigma, k}\left(\omega(\vartheta(z))^{\rho}\right)
$$

respectively.

Theorem 2.1 Let $\rho, \lambda, \delta>0, \omega \in \mathbb{R}, \mathcal{Q}_{1} \in L_{1, r}\left[v_{1}, v_{2}\right]$, and let $\phi_{1}$ and $\phi_{2}$ be two integrable functions defined on $[0, \infty)$ such that

$$
\phi_{1}(x) \leq \mathcal{Q}_{1}(x) \leq \phi_{2}(x)
$$


for all $x \in[0, \infty)$. The we have

$$
\begin{aligned}
& \mathcal{J}_{\rho, \lambda, 0^{+} ; \omega}^{\sigma, k, \phi_{2}}(x) \mathcal{J}_{\rho, \delta, 0^{+} ; \omega}^{\sigma, k, \mathcal{Q}_{1}}(x)+\mathcal{J}_{\rho, \delta, 0^{+} ; \omega}^{\sigma, k, \vartheta} \phi_{1}(x) \mathcal{J}_{\rho, \lambda, 0^{+} ; \omega}^{\sigma, k, \mathcal{Q}_{1}}(x) \\
& \geq \mathcal{J}_{\rho, \delta, 0^{+} ; \omega}^{\sigma, k,} \phi_{1}(x) \mathcal{J}_{\rho, \lambda, 0^{+} ; \omega}^{\sigma, k, \varphi_{2}} \phi_{2}(x)+\mathcal{J}_{\rho, \delta, 0^{+} ; \omega}^{\sigma, k, \vartheta} \mathcal{Q}_{1}(x) \mathcal{J}_{\rho, \lambda, 0^{+} ; \omega}^{\sigma, k, \vartheta} \mathcal{Q}_{1}(x) .
\end{aligned}
$$

Proof Let $t, \eta \in[0, \infty)$. Then from inequality (2.3) it follows that

$$
\left(\phi_{2}(t)-\mathcal{Q}_{1}(t)\right)\left(\mathcal{Q}_{1}(\eta)-\phi_{1}(\eta)\right) \geq 0
$$

which implies that

$$
\phi_{2}(t) \mathcal{Q}_{1}(\eta)+\phi_{1}(\eta) \mathcal{Q}_{1}(t) \geq \phi_{1}(\eta) \phi_{2}(t)+\mathcal{Q}_{1}(\eta) \mathcal{Q}_{1}(t)
$$

Multiplying both sides of (2.6) by

$$
\frac{\vartheta^{\prime}(t) \vartheta^{\prime}(\eta)}{(\vartheta(x)-\vartheta(t))^{1-\frac{\lambda}{k}}(\vartheta(x)-\vartheta(\eta))^{1-\frac{\delta}{k}}} \mathcal{F}_{\rho, \lambda}^{\sigma, k}\left[\omega(\vartheta(x)-\vartheta(\eta))^{\rho}\right] \mathcal{F}_{\rho, \delta}^{\sigma, k}\left[\omega(\vartheta(x)-\vartheta(\eta))^{\rho}\right]
$$

and integrating the obtained inequality with respect to $t$ and $\eta$ over $(0, x)$ give the desired inequality (2.4).

Lemma 2.2 If all the conditions of Theorem 2.1 are satisfied, then we have the equality

$$
\begin{aligned}
{[} & \left.\phi_{2} \mathcal{A}_{\lambda}(x)-\mathcal{J}_{\rho, \lambda, 0^{+} ; \omega}^{\sigma, k,} \mathcal{Q}_{1}(x)\right]\left[\mathcal{J}_{\rho, \lambda, 0^{+} ; \omega}^{\sigma, k, \mathcal{Q}_{1}}(x)-\phi_{1} \mathcal{A}_{\lambda}(x)\right] \\
& -\mathcal{J}_{\rho, \lambda, 0^{+} ; \omega}^{\sigma, k,}\left[\left(\phi_{2}-\mathcal{Q}_{1}(x)\right)\left(\mathcal{Q}_{1}(x)-\phi_{1}\right)\right] \mathcal{A}_{\lambda}(x) \\
= & \mathcal{J}_{\rho, \lambda, 0^{+} ; \omega}^{\sigma, k, \mathcal{Q}_{1}^{2}}(x) \mathcal{A}_{\lambda}(x)-\mathcal{J}_{\rho, \lambda, 0^{+} ; \omega}^{\sigma, k, \vartheta} \mathcal{Q}_{1}(x) \mathcal{J}_{\rho, \lambda, 0^{+} ; \omega}^{\sigma, k, \vartheta} \mathcal{Q}_{1}(x)
\end{aligned}
$$

where $\mathcal{A}_{\lambda}(x)$ is defined by $(2.1)$.

Proof Let $\phi_{1}, \phi_{2} \in \mathbb{R}$, and let $\mathcal{Q}_{1}$ be a function defined on $[0, \infty)$. Then for any $t>0$ and $\eta>0$, we have

$$
\begin{aligned}
\left(\phi_{2}-\right. & \left.\mathcal{Q}_{1}(\eta)\right)\left(\mathcal{Q}_{1}(t)-\phi_{1}\right)+\left(\phi_{2}-\mathcal{Q}_{1}(t)\right)\left(\mathcal{Q}_{1}(\eta)-\phi_{1}\right) \\
& -\left(\phi_{2}-\mathcal{Q}_{1}(t)\right)\left(\mathcal{Q}_{1}(t)-\phi_{1}\right)-\left(\phi_{2}-\mathcal{Q}_{1}(\eta)\right)\left(\mathcal{Q}_{1}(\eta)-\phi_{1}\right) \\
= & \mathcal{Q}_{1}^{2}(t)+\mathcal{Q}_{1}^{2}(\eta)-2 \mathcal{Q}_{1}(t) \mathcal{Q}_{1}(\eta)
\end{aligned}
$$

Multiplying both sides of (2.8) by

$$
\frac{\vartheta^{\prime}(t)}{(\vartheta(x)-\vartheta(t))^{1-\frac{\lambda}{k}}} \mathcal{F}_{\rho, \lambda}^{\sigma, k}\left[\omega(\vartheta(x)-\vartheta(t))^{\rho}\right]
$$

and integrating the obtained result with respect to $t$ over $(0, x)$ lead to

$$
\begin{aligned}
& \left(\phi_{2}-\mathcal{Q}_{1}(\eta)\right)\left[\mathcal{J}_{\rho, \lambda, 0^{+} ; \omega}^{\sigma, k, \mathcal{Q}_{1}}(x)-\phi_{1} \mathcal{A}_{\lambda}(x)\right]+\left[\phi_{2} \mathcal{A}_{\lambda}(x)-\mathcal{J}_{\rho, \lambda, 0^{+} ; \omega}^{\sigma, k, \vartheta} \mathcal{Q}_{1}(x)\right]\left(\mathcal{Q}_{1}(\eta)-\phi_{1}\right) \\
& -\mathcal{J}_{\rho, \lambda, 0^{+} ; \omega}^{\sigma, k, \vartheta}\left[\left(\phi_{2}-\mathcal{Q}_{1}(x)\right)\left(\mathcal{Q}_{1}(x)-\phi_{1}\right)\right]-\left(\phi_{2}-\mathcal{Q}_{1}(\eta)\right)\left(\mathcal{Q}_{1}(\eta)-\phi_{1}\right) \mathcal{A}_{\lambda}(x) \\
& =\mathcal{J}_{\rho, \lambda, 0^{+} ; \omega}^{\sigma, k, \vartheta} \mathcal{Q}_{1}^{2}(x)+\mathcal{Q}_{1}^{2}(\eta) \mathcal{A}_{\lambda}(x)-2 \mathcal{Q}_{1}(\eta) \mathcal{J}_{\rho, \lambda, 0^{+} ; \omega}^{\sigma, k, \vartheta} \mathcal{Q}_{1}(x) \text {. }
\end{aligned}
$$


Again, multiplying both sides of (2.9) by

$$
\frac{\vartheta^{\prime}(\eta)}{(\vartheta(x)-\vartheta(\eta))^{1-\frac{\lambda}{k}}} \mathcal{F}_{\rho, \lambda}^{\sigma, k}\left[\omega(\vartheta(x)-\vartheta(\eta))^{\rho}\right]
$$

and integrating the obtained result with respect to $\eta$ over $(0, x)$ give

$$
\begin{aligned}
{\left[\phi_{2}\right.} & \left.\mathcal{A}_{\lambda}(x)-\mathcal{J}_{\rho, \lambda, 0^{+} ; \omega}^{\sigma, k, \vartheta} \mathcal{Q}_{1}(x)\right]\left[\mathcal{J}_{\rho, \lambda, 0^{+} ; \omega}^{\sigma, k, \vartheta} \mathcal{Q}_{1}(x)-\phi_{1} \mathcal{A}_{\lambda}(x)\right] \\
& +\left[\phi_{2} \mathcal{A}_{\lambda}(x)-\mathcal{J}_{\rho, \lambda, 0^{+} ; \omega}^{\sigma, k, \vartheta} \mathcal{Q}_{1}(x)\right]\left[\mathcal{J}_{\rho, \lambda, 0^{+} ; \omega}^{\sigma, k, \vartheta} \mathcal{Q}_{1}(x)-\phi_{1} \mathcal{A}_{\lambda}(x)\right] \\
& -\mathcal{J}_{\rho, \lambda, 0^{+} ; \omega}^{\sigma, k, \vartheta}\left[\left(\phi_{2}-\mathcal{Q}_{1}(x)\right)\left(\mathcal{Q}_{1}(x)-\phi_{1}\right)\right] \mathcal{A}_{\lambda}(x) \\
& -\mathcal{J}_{\rho, \lambda, 0^{+} ; \omega}^{\sigma, k, \vartheta}\left[\left(\phi_{2}-\mathcal{Q}_{1}(\eta)\right)\left(\mathcal{Q}_{1}(\eta)-\phi_{1}\right)\right] \mathcal{A}_{\lambda}(x) \\
= & \mathcal{J}_{\rho, \lambda, 0^{+} ; \omega}^{\sigma, k, \vartheta} \mathcal{Q}_{1}^{2}(x) \mathcal{A}_{\lambda}(x)+\mathcal{J}_{\rho, \lambda, 0^{+} ; \omega}^{\sigma, k, \vartheta} \mathcal{Q}_{1}^{2}(x) \mathcal{A}_{\lambda}(x) \\
& -2 \mathcal{J}_{\rho, \lambda, 0^{+} ; \omega}^{\sigma, k, \vartheta} \mathcal{Q}_{1}(x) \mathcal{J}_{\rho, \lambda, 0^{+} ; \omega}^{\sigma, k, \vartheta} \mathcal{Q}_{1}(x),
\end{aligned}
$$

which completes the proof of Lemma 2.2.

Lemma 2.3 Under the assumptions of Theorem 2.1, we have

$$
\begin{aligned}
{\left[\phi_{2}\right.} & \left.\mathcal{A}_{\lambda 2}(x)-\mathcal{J}_{\rho, \delta, 0^{+} ; \omega}^{\sigma, k, \vartheta} \mathcal{Q}_{1}(x)\right]\left[\mathcal{J}_{\rho, \lambda, 0^{+} ; \omega}^{\sigma, k, \vartheta} \mathcal{Q}_{1}(x)-\phi_{1} \mathcal{A}_{\lambda}(x)\right] \\
& +\left[\phi_{2} \mathcal{A}_{\lambda}(x)-\mathcal{J}_{\rho, \lambda, 0^{+} ; \omega}^{\sigma, k, \vartheta} \mathcal{Q}_{1}(x)\right]\left[\mathcal{J}_{\rho, \delta, 0^{+} ; \omega}^{\sigma, k, \vartheta} \mathcal{Q}_{1}(x)-\phi_{1} \mathcal{A}_{\lambda 2}(x)\right] \\
& -\mathcal{J}_{\rho, \lambda, 0^{+} ; \omega}^{\sigma, k, \vartheta}\left[\left(\phi_{2}-\mathcal{Q}_{1}(x)\right)\left(\mathcal{Q}_{1}(x)-\phi_{1}\right)\right] \mathcal{A}_{\lambda}(x) \\
& -\mathcal{J}_{\rho, \delta, 0^{+} ; \omega}^{\sigma, k, \vartheta}\left[\left(\phi_{2}-\mathcal{Q}_{1}(\eta)\right)\left(\mathcal{Q}_{1}(\eta)-\phi_{1}\right)\right] \mathcal{A}_{\delta}(x) \\
= & \mathcal{J}_{\rho, \lambda, 0^{+} ; \omega}^{\sigma, k, \vartheta} \mathcal{Q}_{1}^{2}(x) \mathcal{A}_{\delta}(x)+\mathcal{J}_{\rho, \delta, 0^{+} ; \omega}^{\sigma, k, \vartheta} \mathcal{Q}_{1}^{2}(x) \mathcal{A}_{\lambda}(x) \\
& -2 \mathcal{J}_{\rho, \delta, 0^{+} ; \omega}^{\sigma, k, \vartheta} \mathcal{Q}_{1}(x) \mathcal{J}_{\rho, \lambda, 0^{+} ; \omega}^{\sigma, k, \vartheta} \mathcal{Q}_{1}(x),
\end{aligned}
$$

where $\mathcal{A}_{\lambda}(x)$ and $\mathcal{A}_{\delta}(x)$ are defined by (2.1) and (2.2), respectively.

Proof Multiplying both sides of (2.9) by

$$
\frac{\vartheta^{\prime}(\eta)}{(\vartheta(x)-\vartheta(\eta))^{1-\frac{\delta}{k}}} \mathcal{F}_{\rho, \delta}^{\sigma, k}\left[\omega(\vartheta(x)-\vartheta(\eta))^{\rho}\right]
$$

and integrating the obtained results with respect to $t$ over $(0, x)$ lead to

$$
\begin{aligned}
{\left[\phi_{2} \mathcal{A}_{\lambda 2}(x)-\mathcal{J}_{\rho, \delta, 0^{+} ; \omega}^{\sigma, k, \vartheta} \mathcal{Q}_{1}(x)\right]\left[\mathcal{J}_{\rho, \lambda, 0^{+} ; \omega}^{\sigma, k, \vartheta} \mathcal{Q}_{1}(x)-\phi_{1} \mathcal{A}_{\lambda}(x)\right] } \\
\quad+\left[\phi_{2} \mathcal{A}_{\lambda}(x)-\mathcal{J}_{\rho, \lambda, 0^{+} ; \omega}^{\sigma, k, \vartheta} \mathcal{Q}_{1}(x)\right]\left[\mathcal{J}_{\rho, \delta, 0^{+} ; \omega}^{\sigma, k, \vartheta} \mathcal{Q}_{1}(x)-\phi_{1} \mathcal{A}_{\lambda 2}(x)\right] \\
\quad-\mathcal{J}_{\rho, \lambda, 0^{+} ; \omega}^{\sigma, k, \vartheta}\left[\left(\phi_{2}-\mathcal{Q}_{1}(x)\right)\left(\mathcal{Q}_{1}(x)-\phi_{1}\right)\right] \mathcal{A}_{\lambda}(x) \\
\quad-\mathcal{J}_{\rho, k, 0^{+} ; \omega}^{\sigma, k, \vartheta}\left[\left(\phi_{2}-\mathcal{Q}_{1}(\eta)\right)\left(\mathcal{Q}_{1}(\eta)-\phi_{1}\right)\right] \mathcal{A}_{\delta}(x) \\
=\mathcal{J}_{\rho, \lambda, 0^{+} ; \omega}^{\sigma, k, \vartheta} \mathcal{Q}_{1}^{2}(x) \mathcal{A}_{\delta}(x)+\mathcal{J}_{\rho, \delta, 0^{+} ; \omega}^{\sigma, k, \vartheta} \mathcal{Q}_{1}^{2}(x) \mathcal{A}_{\lambda}(x) \\
\quad-2 \mathcal{J}_{\rho, \delta, 0^{+} ; \omega}^{\sigma, k,} \mathcal{Q}_{1}(x) \mathcal{J}_{\rho, \lambda, 0^{+} ; \omega}^{\sigma, k, \vartheta} \mathcal{Q}_{1}(x),
\end{aligned}
$$

which completes the proof of Lemma 2.3. 
Theorem 2.4 Under the assumptions of Theorem 2.1, we have

$$
\begin{aligned}
& \mathcal{J}_{\rho, \lambda, 0^{+} ; \omega}^{\sigma, k, \vartheta} \phi_{2}(x) \mathcal{J}_{\rho, \delta, 0^{+} ; \omega}^{\sigma, k, \mathcal{Q}_{1}}(x)+\mathcal{J}_{\rho, \delta, 0^{+} ; \omega}^{\sigma, k, \vartheta} \phi_{1}(x) \mathcal{J}_{\rho, \lambda, 0^{+} ; \omega}^{\sigma, k, \vartheta} \mathcal{Q}_{1}(x) \\
& \geq \mathcal{J}_{\rho, \delta, 0^{+} ; \omega}^{\sigma, k, \vartheta} \phi_{1}(x) \mathcal{J}_{\rho, \lambda, 0^{+} ; \omega}^{\sigma, k, \vartheta} \phi_{2}(x)+\mathcal{J}_{\rho, \delta, 0^{+} ; \omega}^{\sigma, k, \vartheta} \mathcal{Q}_{1}(x) \mathcal{J}_{\rho, \lambda, 0^{+} ; \omega}^{\sigma, k, \vartheta} \mathcal{Q}_{1}(x) .
\end{aligned}
$$

Proof Let $t, \eta \in[0, \infty)$. Then from inequality (2.6) it follows that

$$
\phi_{2}(t) \mathcal{Q}_{1}(\eta)+\phi_{1}(\eta) \mathcal{Q}_{1}(t) \geq \phi_{1}(\eta) \phi_{2}(t)+\mathcal{Q}_{1}(\eta) \mathcal{Q}_{1}(t)
$$

Multiplying both sides of (2.14) by

$$
\frac{\vartheta^{\prime}(t) \vartheta^{\prime}(\eta)}{(\vartheta(x)-\vartheta(t))^{1-\frac{\lambda}{k}}(\vartheta(x)-\vartheta(\eta))^{1-\frac{\delta}{k}}} \mathcal{F}_{\rho, \lambda}^{\sigma, k}\left[\omega(\vartheta(x)-\vartheta(\eta))^{\rho}\right] \mathcal{F}_{\rho, \delta}^{\sigma, k}\left[\omega(\vartheta(x)-\vartheta(\eta))^{\rho}\right]
$$

and integrating the obtained result with respect to $t$ and $\eta$ over $(0, x)$ give the desired inequality (2.13).

Corollary 2.5 Let $\rho, \lambda>0$ and $\omega \in \mathbb{R}$, and let $\mathcal{Q}_{1} \in L_{1, r}\left[v_{1}, v_{2}\right]$ be such that

$$
\mathfrak{m} \leq \mathcal{Q}_{1}(x) \leq \mathcal{M}
$$

for all $x \in[0, \infty)$. Then we have the inequality

$$
\begin{aligned}
& \mathcal{M} \mathcal{A}_{\lambda}(x) \mathcal{J}_{\rho, \delta, 0^{+} ; \omega}^{\sigma, k, \mathcal{Q}_{1}}(x)+\mathfrak{m} \mathcal{A}_{\delta}(x) \mathcal{J}_{\rho, \lambda, 0^{+} ; \omega}^{\sigma, k, \mathcal{Q}_{1}}(x) \\
& \quad \geq \mathcal{M} \mathfrak{m} \mathcal{A}_{\lambda}(x) \mathcal{A}_{\delta}(x)+\mathcal{J}_{\rho, \lambda, 0^{+} ; \omega}^{\sigma, k, \mathcal{Q}_{1}}(x) \mathcal{J}_{\rho, \delta, 0^{+} ; \omega}^{\sigma, k, \mathcal{Q}_{1}}(x),
\end{aligned}
$$

where $\mathcal{A}_{\lambda}$ and $\mathcal{A}_{\delta}$ are given by (2.1) and (2.2), respectively.

Proof Let $t, \eta \in[0, \infty)$. Then from inequality (2.15) we clearly see that

$$
\left(\mathcal{M}-\mathcal{Q}_{1}(t)\right)\left(\mathcal{Q}_{1}(\eta)-\mathfrak{m}\right) \geq 0,
$$

which implies that

$$
\mathcal{M} \mathcal{Q}_{1}(\eta)+\mathfrak{m} \mathcal{Q}_{1}(t) \geq \mathcal{M m}+\mathcal{Q}_{1}(\eta) \mathcal{Q}_{1}(t)
$$

Multiplying both sides of (2.17) by

$$
\frac{\vartheta^{\prime}(t) \vartheta^{\prime}(\eta)}{(\vartheta(x)-\vartheta(t))^{1-\frac{\lambda}{k}}(\vartheta(x)-\vartheta(\eta))^{1-\frac{\delta}{k}}} \mathcal{F}_{\rho, \lambda}^{\sigma, k}\left[\omega(\vartheta(x)-\vartheta(\eta))^{\rho}\right] \mathcal{F}_{\rho, \delta}^{\sigma, k}\left[\omega(\vartheta(x)-\vartheta(\eta))^{\rho}\right]
$$

and integrating the obtained result with respect to $t$ and $\eta$ over $(0, x)$ lead to the desired inequality (2.16).

Theorem 2.6 Let $\rho, \lambda, \delta>0, \omega \in \mathbb{R}, \mathcal{Q}_{1}, \mathcal{Q}_{2} \in L_{1, r}\left[v_{1}, v_{2}\right]$, and let $\phi_{1}, \phi_{2}, \psi_{1}$, and $\psi_{2}$ be four integrable functions defined on $[0, \infty)$ such that

$$
\phi_{1}(x) \leq \mathcal{Q}_{1}(x) \leq \phi_{1}(x), \quad \psi_{1}(x) \leq \mathcal{Q}_{2}(x) \leq \psi_{1}(x)
$$


for all $x \in[0, \infty)$. Then we have

$$
\begin{aligned}
\mid & \mathcal{J}_{\rho, \lambda, 0^{+} ; \omega}^{\sigma, k, \mathcal{Q}_{1}} \mathcal{Q}_{2}(x) \mathcal{A}_{\delta}(x)+\mathcal{J}_{\rho, \delta, 0^{+} ; \omega}^{\sigma, k, \vartheta} \mathcal{Q}_{1} \mathcal{Q}_{2}(x) \mathcal{A}_{\lambda}(x) \\
& -\mathcal{J}_{\rho, \lambda, 0^{+} ; \omega}^{\sigma, k, \vartheta} \mathcal{Q}_{1}(x) \mathcal{J}_{\rho, \delta, 0^{+} ; \omega}^{\sigma, k, \mathcal{Q}_{2}}(x)-\mathcal{J}_{\rho, \delta, 0^{+} ; \omega}^{\sigma, k, \vartheta} \mathcal{Q}_{1}(x) \mathcal{J}_{\rho, \lambda, 0^{+} ; \omega}^{\sigma, k,} \mathcal{Q}_{2}(x) \mid \\
\leq & \left(\frac{\mathcal{A}_{\lambda}(x) \mathcal{A}_{\delta}(x)}{2}\right)^{2}\left(\phi_{2}-\phi_{1}\right)\left(\psi_{1}-\psi_{2}\right),
\end{aligned}
$$

where $\mathcal{A}_{\lambda}$ and $\mathcal{A}_{\delta}$ are given in (2.1) and (2.2), respectively.

Proof Let $\mathcal{Q}_{1}$ and $\mathcal{Q}_{2}$ be two functions defined on $[0, \infty)$ satisfying assumption (2.18), and let $\mathcal{H}(t, \eta)$ be defined by

$$
\mathcal{H}(t, \eta)=\left(\mathcal{Q}_{1}(t)-\mathcal{Q}_{1}(\eta)\right)\left(\mathcal{Q}_{2}(t)-\mathcal{Q}_{2}(\eta)\right) \quad(t, \eta>0, x>0)
$$

Multiplying both sides of (2.20) by

$$
\frac{\vartheta^{\prime}(t) \vartheta^{\prime}(\eta)}{(\vartheta(x)-\vartheta(t))^{1-\frac{\lambda}{k}}(\vartheta(x)-\vartheta(\eta))^{1-\frac{\delta}{k}}} \mathcal{F}_{\rho, \lambda}^{\sigma, k}\left[\omega(\vartheta(x)-\vartheta(\eta))^{\rho}\right] \mathcal{F}_{\rho, \delta}^{\sigma, k}\left[\omega(\vartheta(x)-\vartheta(\eta))^{\rho}\right]
$$

and integrating the obtained result with respect to $t$ and $\eta$ over $(0, x)$ give

$$
\begin{aligned}
& \int_{0}^{x} \int_{0}^{x} \frac{\vartheta^{\prime}(t) \vartheta^{\prime}(\eta)}{(\vartheta(x)-\vartheta(t))^{1-\frac{\lambda}{k}}(\vartheta(x)-\vartheta(\eta))^{1-\frac{\delta}{k}}} \\
& \times \mathcal{F}_{\rho, \lambda}^{\sigma, k}\left[\omega(\vartheta(x)-\vartheta(\eta))^{\rho}\right] \mathcal{F}_{\rho, \delta}^{\sigma, k}\left[\omega(\vartheta(x)-\vartheta(\eta))^{\rho}\right] \mathcal{H}(t, \eta) d t d \eta \\
& =\mathcal{J}_{\rho, \lambda, 0^{+} ; \omega}^{\sigma, k, \vartheta} \mathcal{Q}_{1} \mathcal{Q}_{2}(x) \mathcal{A}_{\delta}(x)+\mathcal{J}_{\rho, \delta, 0^{+} ; \omega}^{\sigma, k, \vartheta} \mathcal{Q}_{1} \mathcal{Q}_{2}(x) \mathcal{A}_{\lambda}(x) \\
& -\mathcal{J}_{\rho, \lambda, 0^{+} ; \omega}^{\sigma, k, \mathcal{Q}_{1}}(x) \mathcal{J}_{\rho, \delta, 0^{+} ; \omega}^{\sigma, k, \mathcal{Q}_{2}}(x)-\mathcal{J}_{\rho, \delta, 0^{+} ; \omega}^{\sigma, k, \mathcal{Q}_{1}}(x) \mathcal{J}_{\rho, \lambda, 0^{+} ; \omega}^{\sigma, k, \vartheta} \mathcal{Q}_{2}(x) .
\end{aligned}
$$

Applying the Cauchy-Schwarz inequality, we get

$$
\begin{aligned}
&\left(\int_{0}^{x}\right. \int_{0}^{x} \frac{\vartheta^{\prime}(t) \vartheta^{\prime}(\eta)}{(\vartheta(x)-\vartheta(t))^{1-\frac{\lambda}{k}}(\vartheta(x)-\vartheta(\eta))^{1-\frac{\delta}{k}}} \\
&\left.\quad \times \mathcal{F}_{\rho, \lambda}^{\sigma, k}\left[\omega(\vartheta(x)-\vartheta(\eta))^{\rho}\right] \mathcal{F}_{\rho, \delta}^{\sigma, k}\left[\omega(\vartheta(x)-\vartheta(\eta))^{\rho}\right] \mathcal{H}(t, \eta) d t d \eta\right)^{2} \\
& \leq \int_{0}^{x} \int_{0}^{x} \frac{\vartheta^{\prime}(t) \vartheta^{\prime}(\eta)}{(\vartheta(x)-\vartheta(t))^{1-\frac{\lambda}{k}}(\vartheta(x)-\vartheta(\eta))^{1-\frac{\delta}{k}}} \\
& \quad \times \mathcal{F}_{\rho, \lambda}^{\sigma, k}\left[\omega(\vartheta(x)-\vartheta(\eta))^{\rho}\right] \mathcal{F}_{\rho, \delta}^{\sigma, k}\left[\omega(\vartheta(x)-\vartheta(\eta))^{\rho}\right]\left[\mathcal{Q}_{1}(t)-\mathcal{Q}_{1}(\eta)\right]^{2} d t d \eta \\
& \quad+\int_{0}^{x} \int_{0}^{x} \frac{\vartheta^{\prime}(t) \vartheta^{\prime}(\eta)}{(\vartheta(x)-\vartheta(t))^{1-\frac{\lambda}{k}}(\vartheta(x)-\vartheta(\eta))^{1-\frac{\delta}{k}}} \\
& \quad \times \mathcal{F}_{\rho, \lambda}^{\sigma, k}\left[\omega(\vartheta(x)-\vartheta(\eta))^{\rho}\right] \mathcal{F}_{\rho, \delta}^{\sigma, k}\left[\omega(\vartheta(x)-\vartheta(\eta))^{\rho}\right]\left[\mathcal{Q}_{2}(t)-\mathcal{Q}_{2}(\eta)\right]^{2} d t d \eta
\end{aligned}
$$

From

$$
\left[\mathcal{Q}_{1}(t)-\mathcal{Q}_{1}(\eta)\right]^{2}=\mathcal{Q}_{1}^{2}(t)+\mathcal{Q}_{1}^{2}(\eta)-2 \mathcal{Q}_{1}(t) \mathcal{Q}_{1}(\eta)
$$


it follows that

$$
\begin{aligned}
& \int_{0}^{x} \int_{0}^{x} \frac{\vartheta^{\prime}(t) \vartheta^{\prime}(\eta)}{(\vartheta(x)-\vartheta(t))^{1-\frac{\lambda}{k}}(\vartheta(x)-\vartheta(\eta))^{1-\frac{\delta}{k}}} \\
& \times \mathcal{F}_{\rho, \lambda}^{\sigma, k}\left[\omega(\vartheta(x)-\vartheta(\eta))^{\rho}\right] \mathcal{F}_{\rho, \delta}^{\sigma, k}\left[\omega(\vartheta(x)-\vartheta(\eta))^{\rho}\right]\left[\mathcal{Q}_{1}(t)-\mathcal{Q}_{1}(\eta)\right]^{2} d t d \eta \\
& =\mathcal{J}_{\rho, \lambda, 0^{+} ; \omega}^{\sigma, k, \vartheta} \mathcal{Q}_{1}^{2}(x) \mathcal{A}_{\delta}(x)+\mathcal{J}_{\rho, \delta, 0^{+} ; \omega}^{\sigma, k,} \mathcal{Q}_{1}^{2}(x) \mathcal{A}_{\lambda}(x)-2 \mathcal{J}_{\rho, \lambda, 0^{+} ; \omega}^{\sigma, k, \mathcal{Q}_{1}}(x) \mathcal{J}_{\rho, \delta, 0^{+} ; \omega}^{\sigma, k} \mathcal{Q}_{1}(x) \text {. }
\end{aligned}
$$

Analogously,

$$
\begin{aligned}
& \int_{0}^{x} \int_{0}^{x} \frac{\vartheta^{\prime}(t) \vartheta^{\prime}(\eta)}{(\vartheta(x)-\vartheta(t))^{1-\frac{\lambda}{k}}(\vartheta(x)-\vartheta(\eta))^{1-\frac{\delta}{k}}} \\
& \quad \times \mathcal{F}_{\rho, \lambda}^{\sigma, k}\left[\omega(\vartheta(x)-\vartheta(\eta))^{\rho}\right] \mathcal{F}_{\rho, \delta}^{\sigma, k}\left[\omega(\vartheta(x)-\vartheta(\eta))^{\rho}\right]\left[\mathcal{Q}_{2}(t)-\mathcal{Q}_{2}(\eta)\right]^{2} d t d \eta \\
& \quad=\mathcal{J}_{\rho, \lambda, 0^{+} ; \omega}^{\sigma, k,} \mathcal{Q}_{2}^{2}(x) \mathcal{A}_{\delta}(x)+\mathcal{J}_{\rho, \delta, 0^{+} ; \omega}^{\sigma, k,} \mathcal{Q}_{2}^{2}(x) \mathcal{A}_{\lambda}(x)-2 \mathcal{J}_{\rho, \lambda, 0^{+} ; \omega}^{\sigma, k, \vartheta} \mathcal{Q}_{2}(x) \mathcal{J}_{\rho, \delta, 0^{+} ; \omega}^{\sigma, k, \vartheta} \mathcal{Q}_{2}(x)
\end{aligned}
$$

Using (2.23) and (2.24) in (2.22), we obtain

$$
\begin{aligned}
& \left(\int_{0}^{x} \int_{0}^{x} \frac{\vartheta^{\prime}(t) \vartheta^{\prime}(\eta)}{(\vartheta(x)-\vartheta(t))^{1-\frac{\lambda}{k}}(\vartheta(x)-\vartheta(\eta))^{1-\frac{\delta}{k}}}\right. \\
& \left.\times \mathcal{F}_{\rho, \lambda}^{\sigma, k}\left[\omega(\vartheta(x)-\vartheta(\eta))^{\rho}\right] \mathcal{F}_{\rho, \delta}^{\sigma, k}\left[\omega(\vartheta(x)-\vartheta(\eta))^{\rho}\right]\left[\mathcal{Q}_{2}(t)-\mathcal{Q}_{2}(\eta)\right]^{2} d t d \eta\right)^{2} \\
& \leq\left(\mathcal{J}_{\rho, \lambda, 0^{+} ; \omega}^{\sigma, k, \vartheta} \mathcal{Q}_{1}^{2}(x) \mathcal{A}_{\delta}(x)+\mathcal{J}_{\rho, \delta, 0^{+} ; \omega}^{\sigma, k} \mathcal{Q}_{1}^{2}(x) \mathcal{A}_{\lambda}(x)-2 \mathcal{J}_{\rho, \lambda, 0^{+} ; \omega}^{\sigma, k, \mathcal{Q}_{1}}(x) \mathcal{J}_{\rho, \delta, 0^{+} ; \omega}^{\sigma, k, \mathcal{Q}_{1}}(x)\right) \\
& \times\left(\mathcal{J}_{\rho, \lambda, 0^{+} ; \omega}^{\sigma, k, \vartheta} \mathcal{Q}_{2}^{2}(x) \mathcal{A}_{\delta}(x)+\mathcal{J}_{\rho, \delta, 0^{+} ; \omega}^{\sigma, k, \vartheta} \mathcal{Q}_{2}^{2}(x) \mathcal{A}_{\lambda}(x)\right. \\
& \left.-2 \mathcal{J}_{\rho, \lambda, 0^{+} ; \omega}^{\sigma, k, \vartheta} \mathcal{Q}_{2}(x) \mathcal{J}_{\rho, \delta, 0^{+} ; \omega}^{\sigma, k, \mathcal{Q}} \mathcal{Q}_{2}(x)\right) \text {. }
\end{aligned}
$$

From inequalities (2.21) and (2.25) we obtain

$$
\begin{aligned}
\left(\mathcal{J}_{\rho, \lambda, 0^{+} ; \omega}^{\sigma, k, \vartheta} \mathcal{Q}_{1} \mathcal{Q}_{2}(x) \mathcal{A}_{\delta}(x)+\mathcal{J}_{\rho, \delta, 0^{+} ; \omega}^{\sigma, k, \vartheta} \mathcal{Q}_{1} \mathcal{Q}_{2}(x) \mathcal{A}_{\lambda}(x)\right. \\
\left.\quad-\mathcal{J}_{\rho, \lambda, 0^{+} ; \omega}^{\sigma, k, \mathcal{Q}_{1}}(x) \mathcal{J}_{\rho, \delta, 0^{+} ; \omega}^{\sigma, k, \vartheta} \mathcal{Q}_{2}(x)-\mathcal{J}_{\rho, \delta, 0^{+} ; \omega}^{\sigma, k,} \mathcal{Q}_{1}(x) \mathcal{J}_{\rho, \lambda, 0^{+} ; \omega}^{\sigma, k, \mathcal{Q}_{2}}(x)\right)^{2} \\
\leq\left(\mathcal{J}_{\rho, \lambda, 0^{+} ; \omega}^{\sigma, k, \vartheta} \mathcal{Q}_{1}^{2}(x) \mathcal{A}_{\delta}(x)+\mathcal{J}_{\rho, \delta, 0^{+} ; \omega}^{\sigma, k, \vartheta} \mathcal{Q}_{1}^{2}(x) \mathcal{A}_{\lambda}(x)-2 \mathcal{J}_{\rho, \lambda, 0^{+} ; \omega}^{\sigma, k, \vartheta} \mathcal{Q}_{1}(x) \mathcal{J}_{\rho, \delta, 0^{+} ; \omega}^{\sigma, k, \vartheta} \mathcal{Q}_{1}(x)\right) \\
\quad \times\left(\mathcal{J}_{\rho, \lambda, k, 0^{+} ; \omega}^{\sigma, k} \mathcal{Q}_{2}^{2}(x) \mathcal{A}_{\delta}(x)+\mathcal{J}_{\rho, \delta, 0^{+} ; \omega}^{\sigma, k, \vartheta} \mathcal{Q}_{2}^{2}(x) \mathcal{A}_{\lambda}(x)\right. \\
\left.\quad-2 \mathcal{J}_{\rho, \lambda, 0^{+} ; \omega}^{\sigma, k, \vartheta} \mathcal{Q}_{2}(x) \mathcal{J}_{\rho, \delta, 0^{+} ; \omega}^{\sigma, k, \vartheta} \mathcal{Q}_{2}(x)\right) .
\end{aligned}
$$

From $\left(\phi_{2}-\mathcal{Q}_{1}(x)\right)\left(\mathcal{Q}_{1}(x)-\phi_{1}\right) \geq 0$ and $\left(\psi_{2}-\mathcal{Q}_{2}(x)\right)\left(\mathcal{Q}_{2}(x)-\psi_{1}\right) \geq 0$ it follows that

$$
\mathcal{A}_{\delta}(x) \mathcal{J}_{\rho, \lambda, 0^{+} ; \omega}^{\sigma, k, \vartheta}\left(\phi_{2}-\mathcal{Q}_{1}(x)\right)\left(\mathcal{Q}_{1}(x)-\phi_{1}\right) \geq 0
$$

and

$$
\mathcal{A}_{\lambda}(x) \mathcal{J}_{\rho, \delta, 0^{+} ; \omega}^{\sigma, k}\left(\psi_{2}-\mathcal{Q}_{2}(x)\right)\left(\mathcal{Q}_{2}(x)-\psi_{1}\right) \geq 0
$$


Therefore

$$
\begin{aligned}
& \left(\mathcal{J}_{\rho, \lambda, 0^{+} ; \omega}^{\sigma, k, \mathcal{Q}_{1}^{2}}(x) \mathcal{A}_{\delta}(x)+\mathcal{J}_{\rho, \delta, 0^{+} ; \omega}^{\sigma, k, \mathcal{Q}_{1}^{2}}(x) \mathcal{A}_{\lambda}(x)-2 \mathcal{J}_{\rho, \lambda, 0^{+} ; \omega}^{\sigma, k, \vartheta} \mathcal{Q}_{1}(x) \mathcal{J}_{\rho, \delta, 0^{+} ; \omega}^{\sigma, k, \mathcal{Q}_{1}}(x)\right) \\
& \quad \leq\left(\phi_{2} \mathcal{A}_{\delta}(x)-\mathcal{J}_{\rho, \lambda, 0^{+} ; \omega}^{\sigma, k, \vartheta} \mathcal{Q}_{1}(x)\right)\left(\mathcal{J}_{\rho, \lambda, 0^{+} ; \omega}^{\sigma, k, \vartheta} \mathcal{Q}_{1}(x)-\phi_{1} \mathcal{A}_{\delta}(x)\right)
\end{aligned}
$$

and

$$
\begin{aligned}
& \left(\mathcal{J}_{\rho, \lambda, 0^{+} ; \omega}^{\sigma, k, \vartheta} \mathcal{Q}_{2}^{2}(x) \mathcal{A}_{\delta}(x)+\mathcal{J}_{\rho, \delta, 0^{+} ; \omega}^{\sigma, k, \mathcal{Q}_{2}}(x) \mathcal{A}_{\lambda}(x)-2 \mathcal{J}_{\rho, \lambda, 0^{+} ; \omega}^{\sigma, k, \vartheta} \mathcal{Q}_{2}(x) \mathcal{J}_{\rho, \delta, 0^{+} ; \omega}^{\sigma, k, \mathcal{Q}_{2}}(x)\right) \\
& \leq\left(\psi_{2} \mathcal{A}_{\lambda}(x)-\mathcal{J}_{\rho, \delta, 0^{+} ; \omega}^{\sigma, k, \vartheta} \mathcal{Q}_{2}(x)\right)\left(\mathcal{J}_{\rho, \delta, 0^{+} ; \omega}^{\sigma, k, \vartheta} \mathcal{Q}_{2}(x)-\psi_{1} \mathcal{A}_{\lambda}(x)\right) .
\end{aligned}
$$

Combining (2.26), (2.27), and (2.28) and using of Lemma 2.2, we conclude that

$$
\begin{aligned}
&\left(\mathcal{J}_{\rho, \lambda, 0^{+} ; \omega}^{\sigma, k, \vartheta} \mathcal{Q}_{1} \mathcal{Q}_{2}(x) \mathcal{A}_{\delta}(x)+\mathcal{J}_{\rho, \delta, 0^{+} ; \omega}^{\sigma, k, \vartheta} \mathcal{Q}_{1} \mathcal{Q}_{2}(x) \mathcal{A}_{\lambda}(x)\right. \\
&\left.\quad-\mathcal{J}_{\rho, \lambda, 0^{+} ; \omega}^{\sigma, k, \vartheta} \mathcal{Q}_{1}(x) \mathcal{J}_{\rho, \delta, 0^{+} ; \omega}^{\sigma, k, \vartheta} \mathcal{Q}_{2}(x)-\mathcal{J}_{\rho, \delta, 0^{+} ; \omega}^{\sigma, k, \vartheta} \mathcal{Q}_{1}(x) \mathcal{J}_{\rho, \lambda, 0^{+} ; \omega}^{\sigma, k, \mathcal{Q}_{2}}(x)\right)^{2} \\
& \leq\left(\phi_{2} \mathcal{A}_{\delta}(x)-\mathcal{J}_{\rho, \lambda, 0^{+} ; \omega}^{\sigma, k, \mathcal{Q}_{1}}(x)\right)\left(\mathcal{J}_{\rho, \lambda, 0^{+} ; \omega}^{\sigma, k, \vartheta} \mathcal{Q}_{1}(x)-\phi_{1} \mathcal{A}_{\delta}(x)\right) \\
& \quad \times\left(\psi_{2} \mathcal{A}_{\lambda}(x)-\mathcal{J}_{\rho, \delta, 0^{+} ; \omega}^{\sigma, k, \vartheta} \mathcal{Q}_{2}(x)\right)\left(\mathcal{J}_{\rho, \delta, 0^{+} ; \omega}^{\sigma, k, \vartheta} \mathcal{Q}_{2}(x)-\psi_{1} \mathcal{A}_{\lambda}(x)\right) .
\end{aligned}
$$

By the inequality $4 \mu \nu \leq(\mu+v)^{2}$ we get

$$
\begin{aligned}
& 4\left(\phi_{2} \mathcal{A}_{\delta}(x)-\mathcal{J}_{\rho, \lambda, 0^{+} ; \omega}^{\sigma, k, \vartheta} \mathcal{Q}_{1}(x)\right)\left(\mathcal{J}_{\rho, \lambda, 0^{+} ; \omega}^{\sigma, k, \vartheta} \mathcal{Q}_{1}(x)-\phi_{1} \mathcal{A}_{\delta}(x)\right) \\
& \quad \leq\left(\mathcal{A}_{\delta}(x)\left(\phi_{2}-\phi_{1}\right)\right)^{2}, \\
& 4\left(\psi_{2} \mathcal{A}_{\lambda}(x)-\mathcal{J}_{\rho, \delta, 0^{+} ; \omega}^{\sigma, k, \vartheta} \mathcal{Q}_{2}(x)\right)\left(\mathcal{J}_{\rho, \delta, 0^{+} ; \omega}^{\sigma, k, \vartheta} \mathcal{Q}_{2}(x)-\psi_{1} \mathcal{A}_{\lambda}(x)\right) \\
& \quad \leq\left(\mathcal{A}_{\lambda}(x)\left(\psi_{2}-\psi_{1}\right)\right)^{2} .
\end{aligned}
$$

Therefore the desired inequality (2.19) can be obtained from (2.29) and (2.30).

Theorem 2.7 If all the conditions of Theorem 2.6 are satisfied, then we have the following inequalities:

(i) $\mathcal{J}_{\rho, \lambda, 0^{+} ; \omega}^{\sigma, k, \vartheta} \phi_{2}(x) \mathcal{J}_{\rho, \delta, 0^{+} ; \omega}^{\sigma, k, \vartheta} \mathcal{Q}_{2}(x)+\mathcal{J}_{\rho, \delta, 0^{+} ; \omega}^{\sigma, k, \vartheta} \psi_{1}(x) \mathcal{J}_{\rho, \lambda, 0^{+} ; \omega}^{\sigma, k, \vartheta} \mathcal{Q}_{1}(x)$

$$
\geq \mathcal{J}_{\rho, \delta, 0^{+} ; \omega}^{\sigma, k} \psi_{1}(x) \mathcal{J}_{\rho, \lambda, 0^{+} ; \omega}^{\sigma, k, \phi_{2}} \phi_{2}(x)+\mathcal{J}_{\rho, \delta, 0^{+} ; \omega}^{\sigma, k, \vartheta} \mathcal{Q}_{2}(x) \mathcal{J}_{\rho, \lambda, 0^{+} ; \omega}^{\sigma, k, \mathcal{Q}_{1}}(x),
$$

(ii) $\mathcal{J}_{\rho, \lambda, 0^{+} ; \omega}^{\sigma, k, \vartheta} \psi_{1}(x) \mathcal{J}_{\rho, \delta, 0^{+} ; \omega}^{\sigma, k, \vartheta} \mathcal{Q}_{1}(x)+\mathcal{J}_{\rho, \lambda, 0^{+} ; \omega}^{\sigma, k, \mathcal{Q}_{2}}(x) \mathcal{J}_{\rho, \delta, 0^{+} ; \omega}^{\sigma, k, \phi_{1}(x)}$

$$
\geq \mathcal{J}_{\rho, \lambda, 0^{+} ; \omega}^{\sigma, k, \mathcal{Q}_{2}}(x) \mathcal{J}_{\rho, \delta, 0^{+} ; \omega}^{\sigma, k, \mathcal{Q}_{1}}(x)+\mathcal{J}_{\rho, \lambda, 0^{+} ; \omega}^{\sigma, k, \vartheta} \psi_{1}(x) \mathcal{J}_{\rho, \delta, 0^{+} ; \omega}^{\sigma, k, \vartheta} \phi_{1}(x),
$$

(iii) $\mathcal{J}_{\rho, \lambda, 0^{+} ; \omega}^{\sigma, k, \phi_{2}}(x) \mathcal{J}_{\rho, \delta, 0^{+} ; \omega}^{\sigma, k} \mathcal{Q}_{2}(x)+\mathcal{J}_{\rho, \delta, 0^{+} ; \omega}^{\sigma, k} \mathcal{Q}_{1}(x) \mathcal{J}_{\rho, \delta, 0^{+} ; \omega}^{\sigma, k} \psi_{1}(x)$

$$
\geq \mathcal{J}_{\rho, \lambda, 0^{+} ; \omega}^{\sigma, k, \mathcal{Q}_{1}}(x) \mathcal{J}_{\rho, \delta, 0^{+} ; \omega}^{\sigma, k, \mathcal{Q}_{2}}(x)+\mathcal{J}_{\rho, \delta, 0^{+} ; \omega}^{\sigma, k, \vartheta} \psi_{1}(x) \mathcal{J}_{\rho, \lambda, 0^{+} ; \omega}^{\sigma, k, \vartheta} \phi_{2}(x),
$$

(iv) $\mathcal{J}_{\rho, \lambda, 0^{+} ; \omega}^{\sigma, k, \vartheta} \phi_{1}(x) \mathcal{J}_{\rho, \delta, 0^{+} ; \omega}^{\sigma, k, \mathcal{Q}_{2}}(x)+\mathcal{J}_{\rho, \lambda, 0^{+} ; \omega}^{\sigma, k, \mathcal{Q}_{1}}(x) \mathcal{J}_{\rho, \lambda, 0^{+} ; \omega}^{\sigma, k, \psi_{1}} \psi_{1}(x)$

$$
\geq \mathcal{J}_{\rho, \lambda, 0^{+} ; \omega}^{\sigma, k, \vartheta} \mathcal{Q}_{1}(x) \mathcal{J}_{\rho, \delta, 0^{+} ; \omega}^{\sigma, k, \mathcal{Q}_{2}}(x)+\mathcal{J}_{\rho, \lambda, 0^{+} ; \omega}^{\sigma, k, \vartheta} \phi_{1}(x) \mathcal{J}_{\rho, \delta, 0^{+} ; \omega}^{\sigma, k, \vartheta} \psi_{1}(x) .
$$


Proof We first prove part (i). For $x \in[0, \infty)$, from (2.18) it follows that

$$
\begin{aligned}
& \left(\phi_{2}(t)-\mathcal{Q}_{1}(t)\right)\left(\mathcal{Q}_{2}(\eta)-\psi_{1}(\eta)\right) \geq 0 \\
& \phi_{2}(t) \mathcal{Q}_{2}(\eta)+\psi_{1}(\eta) \mathcal{Q}_{1}(t) \geq \psi_{1}(\eta) \phi_{2}(t)+\mathcal{Q}_{2}(\eta) \mathcal{Q}_{1}(t) .
\end{aligned}
$$

Multiplying both sides of (2.31) by

$$
\frac{\vartheta^{\prime}(t) \vartheta^{\prime}(\eta)}{(\vartheta(x)-\vartheta(t))^{1-\frac{\lambda}{k}}(\vartheta(x)-\vartheta(\eta))^{1-\frac{\delta}{k}}} \mathcal{F}_{\rho, \lambda}^{\sigma, k}\left[\omega(\vartheta(x)-\vartheta(\eta))^{\rho}\right] \mathcal{F}_{\rho, \delta}^{\sigma, k}\left[\omega(\vartheta(x)-\vartheta(\eta))^{\rho}\right]
$$

and integrating the obtained result with respect to $t$ and $\eta$ over $(0, x)$ lead to the desired inequality in part (i).

To prove parts (ii)-(iv), we only need to use the inequalities

$$
\begin{gathered}
\left(\psi_{1}(t)-\mathcal{Q}_{2}(t)\right)\left(\mathcal{Q}_{1}(\eta)-\phi_{1}(\eta)\right) \geq 0, \\
\left(\phi_{2}(t)-\mathcal{Q}_{1}(t)\right)\left(\mathcal{Q}_{2}(\eta)-\psi_{1}(\eta)\right) \leq 0,
\end{gathered}
$$

and

$$
\left(\phi_{1}(t)-\mathcal{Q}_{1}(t)\right)\left(\mathcal{Q}_{2}(\eta)-\psi_{1}(\eta)\right) \leq 0
$$

By adopting a similar procedure as we did in the theorem we can easily derive the following lemma.

Lemma 2.8 Let $\mathfrak{m}, \mathcal{M}, \mathfrak{n}, \mathcal{N} \in[0, \infty), \rho, \lambda, \delta>0, \omega \in \mathbb{R}$, and let $\mathcal{Q}_{1}, \mathcal{Q}_{2} \in L_{1, r}\left[v_{1}, v_{2}\right]$ be such that

$$
\mathfrak{m} \leq \mathcal{Q}_{1}(x) \leq \mathcal{M}, \quad \mathfrak{n} \leq \mathcal{Q}_{2}(x) \leq \mathcal{N}
$$

for all $x \in[0, \infty)$. Then

(i) $\mathcal{M A}_{\lambda}(x) \mathcal{J}_{\rho, \delta, 0^{+} ; \omega}^{\sigma, k, \mathcal{Q}_{2}}(x)+\mathfrak{n} \mathcal{A}_{\delta}(x) \mathcal{J}_{\rho, \lambda, 0^{+} ; \omega}^{\sigma, k, \vartheta} \mathcal{Q}_{1}(x)$

$$
\geq \mathfrak{n} \mathcal{M} \mathcal{A}_{\lambda}(x) \mathcal{A}_{\delta}(x)+\mathcal{J}_{\rho, \delta, 0^{+} ; \omega}^{\sigma, k, \vartheta} \mathcal{Q}_{2}(x) \mathcal{J}_{\rho, \lambda, 0^{+} ; \omega}^{\sigma, k,} \mathcal{Q}_{1}(x),
$$

(ii) $\mathcal{M A}_{\lambda}(x) \mathcal{J}_{\rho, \delta, 0^{+} ; \omega}^{\sigma, k,} \mathcal{Q}_{2}(x)+\mathfrak{n} \mathcal{A}_{\delta}(x) \mathcal{J}_{\rho, \lambda, 0^{+} ; \omega}^{\sigma, k, \mathcal{Q}_{1}}(x)$

$$
\geq \mathcal{J}_{\rho, \lambda, 0^{+} ; \omega}^{\sigma, k, \vartheta} \mathcal{Q}_{1}(x) \mathcal{J}_{\rho, \delta, 0^{+} ; \omega}^{\sigma, k, \vartheta} \mathcal{Q}_{2}(x)+\mathfrak{n} \mathcal{M} \mathcal{A}_{\lambda}(x) \mathcal{A}_{\delta}(x),
$$

(iii) $\mathcal{J}_{\rho, \lambda, 0^{+} ; \omega}^{\sigma, k, \mathcal{Q}_{1}}(x) \mathcal{J}_{\rho, \delta, 0^{+} ; \omega}^{\sigma, k, \mathcal{Q}} \mathcal{Q}_{2}(x)+\mathfrak{n} \mathcal{M} \mathcal{A}_{\lambda}(x) \mathcal{A}_{\delta}(x)$

$$
\geq \mathcal{M} \mathcal{A}_{\lambda}(x) \mathcal{J}_{\rho, \delta, 0^{+} ; \omega}^{\sigma, k, \vartheta} \mathcal{Q}_{2}(x)+\mathfrak{n} \mathcal{A}_{\delta} \mathcal{J}_{\rho, \lambda, 0^{+} ; \omega}^{\sigma, k,} \mathcal{Q}_{1}(x),
$$

(iv) $\mathfrak{m} \mathfrak{n} \mathcal{A}_{\lambda}(x) \mathcal{A}_{\delta}(x)+\mathcal{J}_{\rho, \lambda, 0^{+} ; \omega}^{\sigma, k, \mathcal{Q}_{1}}(x) \mathcal{J}_{\rho, \delta, 0^{+} ; \omega}^{\sigma, k, \vartheta} \mathcal{Q}_{2}(x)$

$$
\geq \mathfrak{m} \mathcal{J}_{\rho, \delta, 0^{+} ; \omega}^{\sigma, k, \vartheta} \mathcal{Q}_{2}(x)+\mathfrak{n} \mathcal{J}_{\rho, \lambda, 0^{+} ; \omega}^{\sigma, k, \mathcal{Q}_{1}}(x),
$$

where $\mathcal{A}_{\lambda}$ and $\mathcal{A}_{\delta}$ are given in (2.1) and (2.2), respectively. 


\section{Certain other associated fractional integral inequalities}

Theorem 3.1 Let $\alpha, \beta>1$ with $\alpha^{-1}+\beta^{-1}=1$, and let $\mathcal{Q}_{1}, \mathcal{Q}_{2} \in L_{1, r}\left[v_{1}, v_{2}\right]$. Then we have the inequalities

$$
\begin{aligned}
& \text { (a) } \quad \alpha^{-1} \mathcal{A}_{\delta}(x) \mathcal{J}_{\rho, \lambda, 0^{+} ; \omega}^{\sigma, k, \mathcal{Q}_{1}^{\alpha}}(x)+\beta^{-1} \mathcal{A}_{\lambda}(x) \mathcal{J}_{\rho, \delta, 0^{+} ; \omega}^{\sigma, k} \mathcal{Q}_{2}^{\beta}(x) \\
& \geq \mathcal{J}_{\rho, \lambda, 0^{+} ; \omega}^{\sigma, k, \vartheta} \mathcal{Q}_{1}(x) \mathcal{J}_{\rho, \delta, 0^{+} ; \omega}^{\sigma, k, \vartheta} \mathcal{Q}_{2}(x), \\
& \text { (b) } \quad \alpha^{-1} \mathcal{J}_{\rho, \lambda, 0^{+} ; \omega}^{\sigma, k, \vartheta} \mathcal{Q}_{1}^{\alpha}(x) \mathcal{J}_{\rho, \delta, 0^{+} ; \omega}^{\sigma, k, \vartheta} \mathcal{Q}_{2}^{\alpha}(x)+\beta^{-1} \mathcal{J}_{\rho, \lambda, 0^{+} ; \omega}^{\sigma, k, \vartheta} \mathcal{Q}_{2}^{\beta}(x) \mathcal{J}_{\rho, \delta, 0^{+} ; \omega}^{\sigma, k, \vartheta} \mathcal{Q}_{1}^{\beta}(x) \\
& \geq \mathcal{J}_{\rho, \lambda, 0^{+} ; \omega}^{\sigma, k, \vartheta} \mathcal{Q}_{1} \mathcal{Q}_{2}(x) \mathcal{J}_{\rho, \delta, 0^{+} ; \omega}^{\sigma, k, \vartheta} \mathcal{Q}_{1} \mathcal{Q}_{2}(x), \\
& \text { (c) } \alpha^{-1} \mathcal{J}_{\rho, \lambda, 0^{+} ; \omega}^{\sigma, k, \vartheta} \mathcal{Q}_{1}^{\alpha}(x) \mathcal{J}_{\rho, \delta, 0^{+} ; \omega}^{\sigma, k, \vartheta} \mathcal{Q}_{2}^{\beta}(x)+\beta^{-1} \mathcal{J}_{\rho, \delta, 0^{+} ; \omega}^{\sigma, k, \vartheta} \mathcal{Q}_{1}^{\beta}(x) \mathcal{J}_{\rho, \lambda, 0^{+} ; \omega}^{\sigma, k, \vartheta} \mathcal{Q}_{2}^{\alpha}(x) \\
& \geq \mathcal{J}_{\rho, \lambda, 0^{+} ; \omega}^{\sigma, k, \vartheta} \mathcal{Q}_{1} \mathcal{Q}_{2}^{\alpha-1}(x) \mathcal{J}_{\rho, \delta, 0^{+} ; \omega}^{\sigma, k,} \mathcal{Q}_{1} \mathcal{Q}_{2}^{\beta-1}(x), \\
& \text { (d) } \alpha^{-1} \mathcal{J}_{\rho, \delta, 0^{+} ; \omega}^{\sigma, k, \vartheta} \mathcal{Q}_{1}^{\alpha}(x) \mathcal{J}_{\rho, \lambda, 0^{+} ; \omega}^{\sigma, k, \vartheta} \mathcal{Q}_{2}^{\beta}(x)+\beta^{-1} \mathcal{J}_{\rho, \delta, 0^{+} ; \omega}^{\sigma, k, \vartheta} \mathcal{Q}_{2}^{\beta}(x) \mathcal{J}_{\rho, \lambda, 0^{+} ; \omega}^{\sigma, k, \vartheta} \mathcal{Q}_{1}^{\alpha}(x) \\
& \geq \mathcal{J}_{\rho, \lambda, 0^{+} ; \omega}^{\sigma, k, \vartheta} \mathcal{Q}_{1}^{\alpha-1} \mathcal{Q}_{2}^{\beta-1}(x) \mathcal{J}_{\rho, \delta, 0^{+} ; \omega}^{\sigma, k, \vartheta} \mathcal{Q}_{1} \mathcal{Q}_{2}(x) \\
& \text { (e) } \alpha^{-1} \mathcal{J}_{\rho, \lambda, 0^{+} ; \omega}^{\sigma, k, \vartheta} \mathcal{Q}_{1}^{\alpha}(x) \mathcal{J}_{\rho, \delta, 0^{+} ; \omega}^{\sigma, k,} \mathcal{Q}_{2}^{2}(x)+\beta^{-1} \mathcal{J}_{\rho, \lambda, 0^{+} ; \omega}^{\sigma, k, \vartheta} \mathcal{Q}_{2}^{\beta}(x) \mathcal{J}_{\rho, \delta, 0^{+} ; \omega}^{\sigma, k, \vartheta} \mathcal{Q}_{1}(x) \\
& \geq \mathcal{J}_{\rho, \lambda, 0^{+} ; \omega}^{\sigma, k, \vartheta} \mathcal{Q}_{1} \mathcal{Q}_{2}(x) \mathcal{J}_{\rho, \delta, 0^{+} ; \omega}^{\sigma, k, \vartheta} \mathcal{Q}_{1}^{2 / \beta} \mathcal{Q}_{2}^{2 / \alpha}(x), \\
& \text { (f) } \quad \alpha^{-1} \mathcal{J}_{\rho, \lambda, 0^{+} ; \omega}^{\sigma, k, \vartheta} \mathcal{Q}_{1}^{2}(x) \mathcal{J}_{\rho, \delta, 0^{+} ; \omega}^{\sigma, k,} \mathcal{Q}_{2}^{\beta}(x)+\beta^{-1} \mathcal{J}_{\rho, \delta, 0^{+} ; \omega}^{\sigma, k} \mathcal{Q}_{1}^{\alpha}(x) \mathcal{J}_{\rho, \lambda, 0^{+} ; \omega}^{\sigma, k, \mathcal{Q}_{2}^{2}(x)} \\
& \geq \mathcal{J}_{\rho, \lambda, 0^{+} ; \omega}^{\sigma, k, \vartheta} \mathcal{Q}_{1}^{2 / \alpha} \mathcal{Q}_{2}^{2 / \beta}(x) \mathcal{J}_{\rho, \delta, 0^{+} ; \omega}^{\sigma, k, \vartheta} \mathcal{Q}_{1}^{\alpha-1} \mathcal{Q}_{2}^{\beta-1} \mid(x), \\
& (g) \quad \alpha^{-1} \mathcal{A}_{\delta}(x) \mathcal{J}_{\rho, \lambda, 0^{+} ; \omega}^{\sigma, k} \mathcal{Q}_{1}^{2} \mathcal{Q}_{2}^{\beta}(x)+\mathcal{A}_{\lambda}(x) \mathcal{J}_{\rho, \delta, 0^{+} ; \omega}^{\sigma, k, \vartheta} \mathcal{Q}_{2}^{\alpha} \mathcal{Q}_{1}^{2}(x) \\
& \geq \mathcal{J}_{\rho, \lambda, 0^{+} ; \omega}^{\sigma, k, \vartheta} \mathcal{Q}_{1}^{2 / \alpha} \mathcal{Q}_{2}^{\beta-1}(x) \mathcal{J}_{\rho, \delta, 0^{+} ; \omega}^{\sigma, k, \vartheta} \mathcal{Q}_{1}^{2 / \beta} \mathcal{Q}_{2}^{\alpha-1}(x) \text {. }
\end{aligned}
$$

Proof By the well-known Young inequality

$$
\alpha^{-1} \mu^{\alpha}+\beta^{-1} v^{\beta} \geq \mu \nu \quad\left(\mu, v \geq 0, \alpha, \beta>1, \alpha^{-1}+\beta^{-1}=1\right),
$$

substituting $\mu=\mathcal{Q}_{1}(t)$ and $v=\mathcal{Q}_{2}(\eta)$ for $\eta, t \geq 0$, we have

$$
\alpha^{-1} \mathcal{Q}_{1}(t)^{\alpha}+\beta^{-1} \mathcal{Q}_{2}(\eta)^{\beta} \geq \mathcal{Q}_{1}(t) \mathcal{Q}_{2}(\eta)
$$

Multiplying both sides of (2.32) by

$$
\frac{\vartheta^{\prime}(t) \vartheta^{\prime}(\eta)}{(\vartheta(x)-\vartheta(t))^{1-\frac{\lambda}{k}}(\vartheta(x)-\vartheta(\eta))^{1-\frac{\delta}{k}}} \mathcal{F}_{\rho, \lambda}^{\sigma, k}\left[\omega(\vartheta(x)-\vartheta(\eta))^{\rho}\right] \mathcal{F}_{\rho, \delta}^{\sigma, k}\left[\omega(\vartheta(x)-\vartheta(\eta))^{\rho}\right]
$$

leads to the inequality

$$
\begin{aligned}
& \alpha^{-1} \frac{\vartheta^{\prime}(t) \vartheta^{\prime}(\eta)}{(\vartheta(x)-\vartheta(t))^{1-\frac{\lambda}{k}}(\vartheta(x)-\vartheta(\eta))^{1-\frac{\delta}{k}}} \\
& \quad \times \mathcal{F}_{\rho, \lambda}^{\sigma, k}\left[\omega(\vartheta(x)-\vartheta(\eta))^{\rho}\right] \mathcal{F}_{\rho, \delta}^{\sigma, k}\left[\omega(\vartheta(x)-\vartheta(\eta))^{\rho}\right] \mathcal{Q}_{1}^{\alpha}(t) \\
& \quad+\beta^{-1} \frac{\vartheta^{\prime}(t) \vartheta^{\prime}(\eta)}{(\vartheta(x)-\vartheta(t))^{1-\frac{\lambda}{k}}(\vartheta(x)-\vartheta(\eta))^{1-\frac{\delta}{k}}} \\
& \quad \times \mathcal{F}_{\rho, \lambda}^{\sigma, k}\left[\omega(\vartheta(x)-\vartheta(\eta))^{\rho}\right] \mathcal{F}_{\rho, \delta}^{\sigma, k}\left[\omega(\vartheta(x)-\vartheta(\eta))^{\rho}\right] \mathcal{Q}_{2}^{\beta}(\eta)
\end{aligned}
$$




$$
\begin{aligned}
\geq & \frac{\vartheta^{\prime}(t) \vartheta^{\prime}(\eta)}{(\vartheta(x)-\vartheta(t))^{1-\frac{\lambda}{k}}(\vartheta(x)-\vartheta(\eta))^{1-\frac{\delta}{k}}} \\
& \times \mathcal{F}_{\rho, \lambda}^{\sigma, k}\left[\omega(\vartheta(x)-\vartheta(\eta))^{\rho}\right] \mathcal{F}_{\rho, \delta}^{\sigma, k}\left[\omega(\vartheta(x)-\vartheta(\eta))^{\rho}\right] \mathcal{Q}_{1}(t) \mathcal{Q}_{2}(\eta) .
\end{aligned}
$$

Integrating this inequality with respect to $t$ and $\eta$ over $(0, x)$ gives

$$
\begin{aligned}
& \alpha^{-1} \mathcal{A}_{\delta}(x) \mathcal{J}_{\rho, \lambda, 0^{+} ; \omega}^{\sigma, k, \mathcal{Q}_{1}^{\alpha}}(x)+\beta^{-1} \mathcal{A}_{\lambda}(x) \mathcal{J}_{\rho, \delta, 0^{+} ; \omega}^{\sigma, k,} \mathcal{Q}_{2}^{\beta}(x) \\
& \geq \mathcal{J}_{\rho, \lambda, 0^{+} ; \omega}^{\sigma, k, \mathcal{Q}_{1}}(x) \mathcal{J}_{\rho, \delta, 0^{+} ; \omega}^{\sigma, k, \vartheta} \mathcal{Q}_{2}(x)
\end{aligned}
$$

which implies part (a).

The remaining inequalities (b)-(g) can be proved by using similar arguments and choosing different parameters $\mu$ and $v$ in the Young inequality as follows:

(b) $\quad \mu=\mathcal{Q}_{1}(t) \mathcal{Q}_{2}(\eta), \quad v=\mathcal{Q}_{1}(\eta) \mathcal{Q}_{2}(t)$,

(c) $\quad \mu=\mathcal{Q}_{1}(t) / \mathcal{Q}_{2}(t), \quad v=\mathcal{Q}_{1}(\eta) / \mathcal{Q}_{2}(\eta) \quad\left(\mathcal{Q}_{2}(t) \mathcal{Q}_{2}(\eta) \neq 0\right)$,

(d) $\quad \mu=\mathcal{Q}_{1}(\eta) / \mathcal{Q}_{1}(t), \quad v=\mathcal{Q}_{2}(\eta) / \mathcal{Q}_{2}(t) \quad\left(\mathcal{Q}_{1}(t) \mathcal{Q}_{2}(\eta) \neq 0\right)$,

(e) $\quad \mu=\mathcal{Q}_{1}(t) \mathcal{Q}_{2}^{2 / \alpha}(\eta), \quad \nu=\mathcal{Q}_{1}^{2 / \beta}(\eta) \mathcal{Q}_{2}(t)$,

(f) $\quad \mu=\mathcal{Q}_{1}^{2 / \alpha}(t) / \mathcal{Q}_{1}(\eta), \quad v=\mathcal{Q}_{2}^{2 / \beta}(t) / \mathcal{Q}_{2}(\eta) \quad\left(\mathcal{Q}_{1}(\eta) \mathcal{Q}_{2}(\eta) \neq 0\right)$,

(g) $\quad \mu=\mathcal{Q}_{1}^{2 / \alpha}(t) / \mathcal{Q}_{2}(\eta), \quad v=\mathcal{Q}_{1}^{2 / \beta}(\eta) / \mathcal{Q}_{2}(t) \quad\left(\mathcal{Q}_{2}(t) \mathcal{Q}_{2}(\eta) \neq 0\right)$.

Theorem 3.2 Let $\mathcal{Q}_{1}$ and $\mathcal{Q}_{2}$ be two positive functions defined on $[0, \infty)$ such that

$$
\mathfrak{m}=\min _{0 \leq t \leq x} \frac{\mathcal{Q}_{1}(t)}{\mathcal{Q}_{2}(t)}, \quad \mathcal{M}=\max _{0 \leq t \leq x} \frac{\mathcal{Q}_{1}(t)}{\mathcal{Q}_{2}(t)} .
$$

Then we have

$$
\begin{aligned}
& \text { (a) } \quad 0 \leq \mathcal{J}_{\rho, \lambda, 0^{+} ; \omega}^{\sigma, k, \vartheta} \mathcal{Q}_{1}^{2}(x) \mathcal{J}_{\rho, \lambda, 0^{+} ; \omega}^{\sigma, k, \vartheta} \mathcal{Q}_{2}^{2}(x) \leq \frac{(\mathfrak{m}+\mathcal{M})^{2}}{4 \mathfrak{m} \mathcal{M}}\left(\mathcal{J}_{\rho, \lambda, 0^{+} ; \omega}^{\sigma, k, \vartheta} \mathcal{Q}_{1} \mathcal{Q}_{2}(x)\right)^{2}, \\
& \text { (b) } \quad 0 \leq \sqrt{\mathcal{J}_{\rho, \lambda, 0^{+} ; \omega}^{\sigma, k, \mathcal{Q}_{1}^{2}}(x) \mathcal{J}_{\rho, \lambda, 0^{+} ; \omega}^{\sigma, k, \vartheta} \mathcal{Q}_{2}^{2}(x)}-\mathcal{J}_{\rho, \lambda, 0^{+} ; \omega}^{\sigma, k, \vartheta} \mathcal{Q}_{1} \mathcal{Q}_{2}(x) \\
& \leq \frac{(\sqrt{\mathcal{M}}-\sqrt{\mathfrak{m}})^{2}}{2 \sqrt{\mathfrak{m} \mathcal{M}}}\left(\mathcal{J}_{\rho, \lambda, 0^{+} ; \omega}^{\sigma, k, \vartheta} \mathcal{Q}_{1} \mathcal{Q}_{2}(x)\right), \\
& \text { (c) } \quad 0 \leq \mathcal{J}_{\rho, \lambda, 0^{+} ; \omega}^{\sigma, k, \vartheta} \mathcal{Q}_{1}^{2}(x) \mathcal{J}_{\rho, \lambda, 0^{+} ; \omega}^{\sigma, k, \vartheta} \mathcal{Q}_{2}^{2}(x)-\left(\mathcal{J}_{\rho, \lambda, 0^{+} ; \omega}^{\sigma, k, \vartheta} \mathcal{Q}_{1} \mathcal{Q}_{2}(x)\right)^{2} \\
& \leq \frac{(\mathcal{M}-\mathfrak{m})^{2}}{4 \mathfrak{m} \mathcal{M}}\left(\mathcal{J}_{\rho, \lambda, 0^{+} ; \omega}^{\sigma, k, \vartheta} \mathcal{Q}_{1} \mathcal{Q}_{2}(x)\right)^{2} .
\end{aligned}
$$

Proof It follows from (3.3) that

$$
\left(\frac{\mathcal{Q}_{1}(t)}{\mathcal{Q}_{2}(t)}-\mathfrak{m}\right)\left(\mathcal{M}-\frac{\mathcal{Q}_{1}(t)}{\mathcal{Q}_{2}(t)}\right) \mathcal{Q}_{2}^{2}(t) \geq 0 \quad(0 \leq t \leq x) .
$$

Multiplying both sides of (3.4) by

$$
\frac{\vartheta^{\prime}(t)}{(\vartheta(x)-\vartheta(t))^{1-\frac{\lambda}{k}}} \mathcal{F}_{\rho, \lambda}^{\sigma, k}\left[\omega(\vartheta(x)-\vartheta(\eta))^{\rho}\right]
$$


and integrating the obtained result with respect to $t$ over $(0, x)$ lead to the inequlity

$$
\mathcal{J}_{\rho, \lambda, 0^{+} ; \omega}^{\sigma, \vartheta} \mathcal{Q}_{1}^{2}(x)+\mathfrak{m} \mathcal{M} \mathcal{J}_{\rho, \lambda, 0^{+} ; \omega}^{\sigma, k, \vartheta} \mathcal{Q}_{2}^{2}(x) \leq(\mathfrak{m}+\mathcal{M}) \mathcal{J}_{\rho, \lambda, 0^{+} ; \omega}^{\sigma, k, \vartheta} \mathcal{Q}_{1} \mathcal{Q}_{2}(x)
$$

It follows from $\mathfrak{m} \mathcal{M}>0$ and $\left(\sqrt{\mathcal{J}_{\rho, \lambda, 0^{+} ; \omega}^{\sigma, k, \vartheta} \mathcal{Q}_{1}^{2}(x)}-\sqrt{\left.\mathfrak{m} \mathcal{M} \mathcal{J}_{\rho, \lambda, 0^{+} ; \omega}^{\sigma, k, \mathcal{Q}_{2}^{2}(x)}\right)^{2}} \geq 0\right.$ that

$$
2 \sqrt{\mathcal{J}_{\rho, \lambda, 0^{+} ; \omega}^{\sigma, k, \vartheta} \mathcal{Q}_{1}^{2}(x)} \sqrt{\mathfrak{m} \mathcal{M} \mathcal{J}_{\rho, \lambda, 0^{+} ; \omega}^{\sigma, k, \vartheta} \mathcal{Q}_{2}^{2}(x)} \leq \mathcal{J}_{\rho, \lambda, 0^{+} ; \omega}^{\sigma, k, \vartheta} \mathcal{Q}_{1}^{2}(x)+\mathfrak{m} \mathcal{M J}_{\rho, \lambda, 0^{+} ; \omega}^{\sigma, k, \vartheta} \mathcal{Q}_{2}^{2}(x)
$$

From (3.5)and (3.6) we clearly see that

$$
4 \mathfrak{m} \mathcal{M}_{\rho, \lambda, 0^{+} ; \omega}^{\sigma, k, \vartheta} \mathcal{Q}_{1}^{2}(x) \mathcal{J}_{\rho, \lambda, 0^{+} ; \omega}^{\sigma, k, \vartheta} \mathcal{Q}_{2}^{2}(x) \leq(\mathfrak{m}+\mathcal{M})^{2}\left(\mathcal{J}_{\rho, \lambda, 0^{+} ; \omega}^{\sigma, k, \vartheta} \mathcal{Q}_{1} \mathcal{Q}_{2}(x)\right)^{2}
$$

which completes the proof of part (a). Parts (b) and (c) can be proved by using similar arguments as in part (a).

Theorem 3.3 Let $\rho, \lambda>0, \omega \in \mathbb{R}, \mathcal{Q}_{1}, \mathcal{Q}_{2} \in L_{1, r}\left[v_{1}, v_{2}\right]$, and let $\gamma, \Upsilon, \theta$, and $\Theta$ be four integrable functions defined on $[0, \infty)$ such that

$$
0<\gamma(x) \leq \mathcal{Q}_{1}(x) \leq \Upsilon(x), \quad 0<\theta(x) \leq \mathcal{Q}_{2}(x) \leq \Theta(x)
$$

for all $x \in[0, \infty)$. Then we have

$$
\begin{aligned}
& \text { (a) } 0 \leq \mathcal{J}_{\rho, \lambda, 0^{+} ; \omega}^{\sigma, k, \vartheta} \mathcal{Q}_{1}^{2}(x) \mathcal{J}_{\rho, \lambda, 0^{+} ; \omega}^{\sigma, k, \vartheta} \mathcal{Q}_{2}^{2}(x) \leq \frac{(\gamma \theta+\Upsilon \Theta)^{2}}{4 \gamma \theta \Upsilon \Theta}\left(\mathcal{J}_{\rho, \lambda, 0^{+} ; \omega}^{\sigma, k, \vartheta} \mathcal{Q}_{1} \mathcal{Q}_{2}(x)\right)^{2} \\
& \text { (b) } 0 \leq \sqrt{\mathcal{J}_{\rho, \lambda, 0^{+} ; \omega}^{\sigma, k, \vartheta} \mathcal{Q}_{1}^{2}(x) \mathcal{J}_{\rho, \lambda, 0^{+} ; \omega}^{\sigma, k, \vartheta} \mathcal{Q}_{2}^{2}(x)}-\left(\mathcal{J}_{\rho, \lambda, 0^{+} ; \omega}^{\sigma, k, \vartheta} \mathcal{Q}_{1} \mathcal{Q}_{2}(x)\right) \\
& \leq \frac{(\sqrt{\Upsilon \Theta}-\sqrt{\gamma \theta})^{2}}{2 \sqrt{\gamma \theta \Upsilon \Theta}}\left(\mathcal{J}_{\rho, \lambda, 0^{+} ; \omega}^{\sigma, k, \vartheta} \mathcal{Q}_{1} \mathcal{Q}_{2}(x)\right), \\
& \text { (c) } \quad 0 \leq \mathcal{J}_{\rho, \lambda, 0^{+} ; \omega}^{\sigma, k, \vartheta} \mathcal{Q}_{1}^{2}(x) \mathcal{J}_{\rho, \lambda, 0^{+} ; \omega}^{\sigma, k, \vartheta} \mathcal{Q}_{2}^{2}(x)-\left(\mathcal{J}_{\rho, \lambda, 0^{+} ; \omega}^{\sigma, k, \vartheta} \mathcal{Q}_{1} \mathcal{Q}_{2}(x)\right)^{2} \\
& \leq \frac{(\Upsilon \Theta-\gamma \theta)^{2}}{4 \gamma \theta \Upsilon \Theta}\left(\mathcal{J}_{\rho, \lambda, 0^{+} ; \omega}^{\sigma, k, \vartheta} \mathcal{Q}_{1} \mathcal{Q}_{2}(x)\right)^{2} \text {. }
\end{aligned}
$$

Proof It follows from inequality (3.7) that

$$
\frac{\gamma}{\Theta} \leq \frac{\mathcal{Q}_{1}(t)}{\mathcal{Q}_{2}(t)} \leq \frac{\Upsilon}{\theta}
$$

Applying Theorem 3.3, we obtain part (a), and parts (b) and (c) can be derived from part (a).

Theorem 3.4 Let $s, \mathfrak{m}, \mathcal{M} \in \mathbb{R}$ with $s \neq 0, \rho, \lambda>0$, and $\omega \in \mathbb{R}$, and let $\mathcal{Q}_{1}, \mathcal{Q}_{2} \in L_{1, r}\left[v_{1}, v_{2}\right]$ be such that

$$
0<\mathfrak{m}<\frac{\mathcal{Q}_{2}(x)}{\mathcal{Q}_{1}(x)} \leq \mathcal{M}<\infty
$$


Then we have the inequality

$$
\begin{aligned}
& \mathcal{J}_{\rho, \lambda, 0^{+} ; \omega}^{\sigma, k, \vartheta} \mathcal{Q}_{1}^{2-s} \mathcal{Q}_{2}^{s}(x)+\frac{\mathfrak{m} \mathcal{M}\left(\mathcal{M}^{s-1}-\mathfrak{m}^{s-1}\right)}{\mathcal{M}-\mathfrak{m}} \mathcal{J}_{\rho, \lambda, 0^{+} ; \omega}^{\sigma, k, \vartheta} \mathcal{Q}_{1}^{s}(x) \\
& \quad \leq \frac{\mathcal{M}^{s}-\mathfrak{m}^{s}}{\mathcal{M}-\mathfrak{m}} \mathcal{J}_{\rho, \lambda, 0^{+} ; \omega}^{\sigma, k,} \mathcal{Q}_{1} \mathcal{Q}_{2}(x)
\end{aligned}
$$

for $s \notin(0,1)$. If $s \in(0,1)$, then inequality (3.10) is reversed. Especially, if $s=2$, then we get (3.5).

Proof The theorem can be easily proved by using the Lah-Ribarič inequality $[29,30]$.

Theorem 3.5 Let $\mathcal{Q}_{1}, \mathcal{Q}_{2} \in L_{1, r}\left[v_{1}, v_{2}\right]$, and let $s \neq 0$ be a real number. Then we have the inequality

$$
\left(\mathcal{J}_{\rho, \lambda, 0^{+} ; \omega}^{\sigma, k, \vartheta} \mathcal{Q}_{1} \mathcal{Q}_{2}(x)\right)^{s} \leq\left(\mathcal{J}_{\rho, \lambda, 0^{+} ; \omega}^{\sigma, k, \vartheta} \mathcal{Q}_{1}^{2}(x)\right)^{s-1} \mathcal{J}_{\rho, \lambda, 0^{+} ; \omega}^{\sigma, k, \vartheta} \mathcal{Q}_{1}^{2-s} \mathcal{Q}_{2}^{s}(x)
$$

for $s \in(0,1)$, and inequality $(3.11)$ is reversed if $s \in(0,1)$.

Proof The theorem can be proved by using the Jensen inequality for convex functions.

Theorem 3.6 Let $0<\alpha \leq \beta<1$ with $\alpha+\beta=1, \rho, \lambda>0$, and $\omega \in \mathbb{R}$, and let $\mathcal{G}, \mathcal{Q}_{1}, \mathcal{Q}_{2} \in$ $L_{1, r}\left[v_{1}, v_{2}\right]$ be such that (3.7) is true. Then

$$
\left(\mathcal{J}_{\rho, \lambda, 0^{+} ; \omega}^{\sigma, k, \vartheta} \mathcal{Q}_{1} \mathcal{G}(x)\right)^{\beta}\left(\mathcal{J}_{\rho, \lambda, 0^{+} ; \omega}^{\sigma, k, \vartheta} \frac{\mathcal{G}(x)}{\mathcal{Q}_{1}(x)}\right)^{\alpha} \leq \frac{\alpha \gamma+\beta \Upsilon}{(\gamma \Upsilon)^{\alpha}}\left(\mathcal{J}_{\rho, \lambda, 0^{+} ; \omega}^{\sigma, k, \vartheta} \mathcal{G}(x)\right)
$$

and

$$
\left(\mathcal{J}_{\rho, \lambda, 0^{+} ; \omega}^{\sigma, k, \mathcal{Q}_{1}} \mathcal{G}(x)\right)^{\beta}\left(\mathcal{J}_{\rho, \lambda, 0^{+} ; \omega}^{\sigma, k, \mathcal{G}} \mathcal{G} \mathcal{Q}_{2}(x)\right)^{\alpha} \leq \frac{\alpha \gamma \theta+\beta \Upsilon \Theta}{(\gamma \Upsilon)^{\alpha}(\theta \Theta)^{\beta}}\left(\mathcal{G} \mathcal{Q}_{1} \mathcal{Q}_{2}(x)\right)
$$

Proof It follows from $\left(\beta \mathcal{Q}_{1}(t)-\alpha \gamma\right)\left(\mathcal{Q}_{1}(t)-\Upsilon\right) \leq$ that

$$
\beta \mathcal{Q}_{1}^{2}(t)-(\alpha \gamma+\beta \Upsilon)+\alpha \gamma \Upsilon \leq 0
$$

Multiplying both sides of (3.14) by $\mathcal{G}(t) / \mathcal{Q}_{1}(t)$ leads to

$$
\beta \mathcal{G}(t) \mathcal{Q}_{1}(t)+\alpha \gamma \Upsilon \frac{\mathcal{G}(t)}{\mathcal{Q}_{1}(t)} \leq \mathcal{G}(t)(\alpha \gamma+\beta \Upsilon)
$$

From (3.15) and the arithmetic-geometric mean inequality we obtain

$$
\begin{aligned}
\left(\int_{0}^{t}\right. & \left.\frac{\vartheta^{\prime}(t)}{(\vartheta(x)-\vartheta(t))^{1-\frac{\lambda}{k}}} \mathcal{F}_{\rho, \lambda}^{\sigma, k}\left[\omega(\vartheta(x)-\vartheta(t))^{\rho}\right] \mathcal{Q}_{1}(t) \mathcal{G}(t) d t\right)^{\beta} \\
& \times\left(\int_{0}^{t} \frac{\vartheta^{\prime}(t)}{(\vartheta(x)-\vartheta(t))^{1-\frac{\lambda}{k}}} \mathcal{F}_{\rho, \lambda}^{\sigma, k}\left[\omega(\vartheta(x)-\vartheta(t))^{\rho}\right] \frac{\mathcal{G}(t)}{\mathcal{Q}_{1}(t)} d t\right)^{\alpha} \\
\quad & \frac{1}{(\gamma \Upsilon)^{\alpha}}\left(\int_{0}^{t} \frac{\vartheta^{\prime}(t)}{(\vartheta(x)-\vartheta(t))^{1-\frac{\lambda}{k}}} \mathcal{F}_{\rho, \lambda}^{\sigma, k}\left[\omega(\vartheta(x)-\vartheta(t))^{\rho}\right] \mathcal{Q}_{1}(t) \mathcal{G}(t) d t\right)^{\beta}
\end{aligned}
$$




$$
\begin{aligned}
& \times\left(\gamma \Upsilon \int_{0}^{t} \frac{\vartheta^{\prime}(t)}{(\vartheta(x)-\vartheta(t))^{1-\frac{\lambda}{k}}} \mathcal{F}_{\rho, \lambda}^{\sigma, k}\left[\omega(\vartheta(x)-\vartheta(t))^{\rho}\right] \frac{\mathcal{G}(t)}{\mathcal{Q}_{1}(t)} d t\right)^{\alpha} \\
\leq & \frac{1}{(\gamma \Upsilon)^{\alpha}}\left(\beta \int_{0}^{t} \frac{\vartheta^{\prime}(t)}{(\vartheta(x)-\vartheta(t))^{1-\frac{\lambda}{k}}} \mathcal{F}_{\rho, \lambda}^{\sigma, k}\left[\omega(\vartheta(x)-\vartheta(t))^{\rho}\right] \mathcal{Q}_{1}(t) \mathcal{G}(t) d t\right. \\
& \left.+\alpha \gamma \Upsilon \int_{0}^{t} \frac{\vartheta^{\prime}(t)}{(\vartheta(x)-\vartheta(t))^{1-\frac{\lambda}{k}}} \mathcal{F}_{\rho, \lambda}^{\sigma, k}\left[\omega(\vartheta(x)-\vartheta(t))^{\rho}\right] \frac{\mathcal{G}(t)}{\mathcal{Q}_{1}(t)} d t\right) \\
= & \frac{\alpha \gamma+\beta \Upsilon}{(\gamma \Upsilon)^{\alpha}}\left(\int_{0}^{t} \frac{\vartheta^{\prime}(t)}{(\vartheta(x)-\vartheta(t))^{1-\frac{\lambda}{k}}} \mathcal{F}_{\rho, \lambda}^{\sigma, k}\left[\omega(\vartheta(x)-\vartheta(t))^{\rho}\right] \mathcal{G}(t) d t\right),
\end{aligned}
$$

which gives the required inequality (3.12).

Replacing $\mathcal{G}$ and $\mathcal{Q}_{1}$ by $\mathcal{G} \mathcal{Q}_{1} \mathcal{Q}_{2}$ and $\mathcal{Q}_{1} / \mathcal{Q}_{2}$ in (3.16) and using the inequality $\gamma / \Theta \leq$ $\mathcal{Q}_{1}(t) / \mathcal{Q}_{2}(t) \leq \Upsilon / \theta$, we obtain

$$
\begin{aligned}
& \left(\int_{0}^{t} \frac{\vartheta^{\prime}(t)}{(\vartheta(x)-\vartheta(t))^{1-\frac{\lambda}{k}}} \mathcal{F}_{\rho, \lambda}^{\sigma, k}\left[\omega(\vartheta(x)-\vartheta(t))^{\rho}\right] \mathcal{Q}_{1}(t) \mathcal{G}(t) d t\right)^{\beta} \\
& \quad \times\left(\int_{0}^{t} \frac{\vartheta^{\prime}(t)}{(\vartheta(x)-\vartheta(t))^{1-\frac{\lambda}{k}}} \mathcal{F}_{\rho, \lambda}^{\sigma, k}\left[\omega(\vartheta(x)-\vartheta(t))^{\rho}\right] \mathcal{G}(t) \mathcal{Q}_{2}(t) d t\right)^{\alpha} \\
& \quad \leq \frac{\alpha \gamma \theta+\beta \Upsilon \Theta}{(\gamma \Upsilon)^{\alpha}(\theta \Theta)^{\beta}}\left(\int_{0}^{t} \frac{\vartheta^{\prime}(t)}{(\vartheta(x)-\vartheta(t))^{1-\frac{\lambda}{k}}} \mathcal{F}_{\rho, \lambda}^{\sigma, k}\left[\omega(\vartheta(x)-\vartheta(t))^{\rho}\right] \mathcal{G}(t) \mathcal{Q}_{1}(t) \mathcal{Q}_{2}(t) d t\right)
\end{aligned}
$$

which implies inequality (3.13).

Theorem 3.7 Let $\rho, \lambda>0$ and $\omega \in \mathbb{R}$, and let $\mathcal{G}, \mathcal{Q}_{1}, \mathcal{Q}_{2} \in L_{1, r}\left[v_{1}, v_{2}\right]$ with $\mathcal{G}(t) \geq 0$. Then the following statements are true:

(a) If there exist constants $\gamma, \Upsilon, \theta, \Theta \in \mathbb{R}$ such that $\left(\Upsilon \mathcal{Q}_{2}(t)-\theta \mathcal{Q}_{1}(t)\right)\left(\Theta \mathcal{Q}_{1}(t)-\gamma \mathcal{Q}_{2}(t)\right) \geq$ 0 for $t>0$, then

$$
\begin{aligned}
& \gamma \Upsilon \mathcal{J}_{\rho, \lambda, 0^{+} ; \omega}^{\sigma, k, \mathcal{G Q}_{2}}(x)+\theta \Theta \mathcal{J}_{\rho, \lambda, 0^{+} ; \omega}^{\sigma, k, \mathcal{G}_{1}} \mathcal{Q}_{1}^{2}(x) \leq(\gamma \theta+\Upsilon \Theta) \mathcal{J}_{\rho, \lambda, 0^{+} ; \omega}^{\sigma, k, \vartheta} \mathcal{G} \mathcal{Q}_{1} \mathcal{Q}_{2}(x) \\
& \quad \leq(\gamma \theta+\Upsilon \Theta)\left(\mathcal{J}_{\rho, \lambda, 0^{+} ; \omega}^{\sigma, k, \vartheta} \mathcal{G} \mathcal{Q}_{2}^{2}(x)+\mathcal{J}_{\rho, \lambda, 0^{+} ; \omega}^{\sigma, k, \vartheta} \mathcal{G} \mathcal{Q}_{1}^{2}(x)\right) .
\end{aligned}
$$

Also, if $\gamma \Upsilon \theta \Theta>0$, then

$$
\begin{aligned}
& \sqrt{\frac{\gamma \Upsilon}{\theta \Theta}} \mathcal{J}_{\rho, \lambda, \lambda, 0^{+} ; \omega}^{\sigma, k} \mathcal{G} \mathcal{Q}_{2}^{2}(x)+\sqrt{\frac{\theta \Theta}{\gamma \Upsilon}} \mathcal{J}_{\rho, \lambda, 0^{+} ; \omega}^{\sigma, k, \vartheta} \mathcal{G} \mathcal{Q}_{1}^{2}(x) \\
& \leq\left(\sqrt{\frac{\Theta \Upsilon}{\theta \gamma}}+\sqrt{\frac{\gamma \theta}{\Upsilon \Theta}}\right) \mathcal{J}_{\rho, \lambda, 0^{+} ; \omega}^{\sigma, k, \vartheta} \mathcal{G} \mathcal{Q}_{1} \mathcal{Q}_{2}(x), \\
& \mathcal{J}_{\rho, \lambda, 0^{+} ; \omega}^{\sigma, k, \vartheta} \mathcal{G} \mathcal{Q}_{2}^{2}(x) \mathcal{J}_{\rho, \lambda, 0^{+} ; \omega}^{\sigma, k, \vartheta} \mathcal{G} \mathcal{Q}_{1}^{2}(x) \leq\left(\frac{\theta \gamma+\Theta \Upsilon}{2 \theta \gamma \Upsilon \Theta}\right)^{2} \mathcal{J}_{\rho, \lambda, 0^{+} ; \omega}^{\sigma, k, \vartheta} \mathcal{G} \mathcal{Q}_{1} \mathcal{Q}_{2}(x) .
\end{aligned}
$$

(b) If there exist constants $\gamma, \Upsilon, \theta, \Theta \in \mathbb{R}$ such that $\left(\Upsilon \mathcal{Q}_{2}(t)-\theta \mathcal{Q}_{1}(\eta)\right)\left(\Theta \mathcal{Q}_{1}(\eta)-\right.$ $\left.\gamma \mathcal{Q}_{2}(t)\right) \geq 0$ for $\eta, t>0$, then

$$
\begin{aligned}
& \gamma \Upsilon \mathcal{J}_{\rho, \lambda, 0^{+} ; \omega}^{\sigma, k, \vartheta} \mathcal{Q}_{2}^{2}(x) \mathcal{J}_{\rho, \delta, 0^{+} ; \omega}^{\sigma, k, \vartheta} \mathcal{G}(x)+\theta \Theta \mathcal{J}_{\rho, \lambda, 0^{+} ; \omega}^{\sigma, k, \vartheta} \mathcal{G}(x) \mathcal{J}_{\rho, \delta, 0^{+} ; \omega}^{\sigma, k, \vartheta} \mathcal{G} \mathcal{Q}_{1}^{2}(x) \\
& \quad \leq(\gamma \theta+\Upsilon \Theta) \mathcal{J}_{\rho, \lambda, 0^{+} ; \omega}^{\sigma, k, \vartheta} \mathcal{G} \mathcal{Q}_{2}(x) \mathcal{J}_{\rho, \delta, 0^{+} ; \omega}^{\sigma, k, \vartheta} \mathcal{G} \mathcal{Q}_{1}(x) .
\end{aligned}
$$


(c) If $\gamma, \Upsilon>0$ and $\theta, \Theta>0$, then

$$
\begin{aligned}
& \gamma \Upsilon\left(\mathcal{J}_{\rho, \lambda, 0^{+} ; \omega}^{\sigma, k} \mathcal{G} \mathcal{Q}_{2}(x)\right)^{2}+\theta \Theta\left(\mathcal{J}_{\rho, \lambda, 0^{+} ; \omega}^{\sigma, k, \vartheta} \mathcal{G} \mathcal{Q}_{1}(x)\right)^{2} \\
& \quad \leq(\gamma \theta+\Upsilon \Theta) \mathcal{J}_{\rho, \lambda, 0^{+} ; \omega}^{\sigma, k, \vartheta} \mathcal{G}(x) \mathcal{J}_{\rho, \lambda, 0^{+} ; \omega}^{\sigma, k, \vartheta} \mathcal{G} \mathcal{Q}_{2} \mathcal{Q}_{1}(x) .
\end{aligned}
$$

(d) If $\gamma, \Upsilon>0$ and $\theta, \Theta>0$, then

$$
\begin{aligned}
& \gamma \Upsilon\left(\mathcal{J}_{\rho, \lambda, 0^{+} ; \omega}^{\sigma, k, \vartheta} \mathcal{G} \mathcal{Q}_{2}(x)\right)^{2}+\theta \Theta\left(\mathcal{J}_{\rho, \lambda, 0^{+} ; \omega}^{\sigma, k, \vartheta} \mathcal{G} \mathcal{Q}_{1}(x)\right)^{2} \\
& \leq(\gamma \theta+\Upsilon \Theta) \mathcal{J}_{\rho, \lambda, 0^{+} ; \omega}^{\sigma, k, \vartheta} \mathcal{G} \mathcal{Q}_{1}(x) \mathcal{J}_{\rho, \lambda, 0^{+} ; \omega}^{\sigma, k, \vartheta} \mathcal{G} \mathcal{Q}_{2}(x) .
\end{aligned}
$$

Proof We first prove part (a). It follows from the assumption that

$$
\mathcal{G}(t)\left(\Upsilon \mathcal{Q}_{2}(t)-\theta \mathcal{Q}_{1}(t)\right)\left(\Theta \mathcal{Q}_{1}(t)-\gamma \mathcal{Q}_{2}(t)\right) \geq 0
$$

for all $t \geq 0$, which implies that

$$
\gamma \Upsilon \mathcal{G}(t) \mathcal{Q}_{2}^{2}(t)+\theta \Theta \mathcal{G}(t) \mathcal{Q}_{1}^{2}(t) \leq(\gamma \theta+\Upsilon \Theta) \mathcal{G}(t) \mathcal{Q}_{1}(t) \mathcal{Q}_{2}(t)
$$

Multiplying both sides of (3.23) by

$$
\frac{\vartheta^{\prime}(t)}{(\vartheta(x)-\vartheta(t))^{1-\frac{\lambda}{k}}} \mathcal{F}_{\rho, \lambda}^{\sigma, k}\left[\omega(\vartheta(x)-\vartheta(\eta))^{\rho}\right]
$$

and integrating the obtained result with respect to $t$ over $(0, x)$ give the left-hand side of (3.17).

Moreover, by Cauchy's inequality we obtain the right-side of (3.17). Multiplying both sides of the inequality

$$
\begin{aligned}
\gamma & \Upsilon \mathcal{J}_{\rho, \lambda, 0^{+} ; \omega}^{\sigma, k, \vartheta} \mathcal{G} \mathcal{Q}_{2}^{2}(x)+\theta \Theta \mathcal{J}_{\rho, \lambda, 0^{+} ; \omega}^{\sigma, k, \vartheta} \mathcal{G} \mathcal{Q}_{1}^{2}(x) \\
& \leq(\gamma \theta+\Upsilon \Theta) \mathcal{J}_{\rho, \lambda, 0^{+} ; \omega}^{\sigma, k, \vartheta} \mathcal{G} \mathcal{Q}_{1} \mathcal{Q}_{2}(x) \\
& \leq(\gamma \theta+\Upsilon \Theta)\left(\mathcal{J}_{\rho, \lambda, 0^{+} ; \omega}^{\sigma, k, \vartheta} \mathcal{G} \mathcal{Q}_{2}^{2}(x)+\mathcal{J}_{\rho, \lambda, 0^{+} ; \omega}^{\sigma, k, \vartheta} \mathcal{G} \mathcal{Q}_{1}^{2}(x)\right)
\end{aligned}
$$

by $1 / \sqrt{\gamma \theta \Upsilon \Theta}$, we get (3.18).

On the other hand, it follows from $\gamma \theta \Upsilon \Theta>0$ and

$$
\left(\sqrt{\gamma \Upsilon \mathcal{J}_{\rho, \lambda, 0^{+} ; \omega}^{\sigma, k, \vartheta} \mathcal{G} \mathcal{Q}_{2}^{2}(x)}-\sqrt{\theta \Theta \mathcal{J}_{\rho, \lambda, 00^{+} ; \omega}^{\sigma, k, \vartheta} \mathcal{G} \mathcal{Q}_{1}^{2}(x)}\right)^{2} \geq 0
$$

that

$$
\begin{aligned}
& 2 \sqrt{\gamma \Upsilon \mathcal{J}_{\rho, \lambda, 0^{+} ; \omega}^{\sigma, k, \vartheta} \mathcal{G} \mathcal{Q}_{2}^{2}(x)} \sqrt{\theta \Theta \mathcal{J}_{\rho, \lambda, 0^{+} ; \omega}^{\sigma, k, \vartheta} \mathcal{G} \mathcal{Q}_{1}^{2}(x)} \\
& \quad \leq \gamma \Upsilon \mathcal{J}_{\rho, \lambda, 0^{+} ; \omega}^{\sigma, k, \vartheta} \mathcal{G} \mathcal{Q}_{2}^{2}(x)+\theta \Theta \mathcal{J}_{\rho, \lambda, 0^{+} ; \omega}^{\sigma, k, \vartheta} \mathcal{G} \mathcal{Q}_{1}^{2}(x) .
\end{aligned}
$$

According (3.24) and (3.25), we have

$$
4 \gamma \Upsilon \theta \Theta \mathcal{J}_{\rho, \lambda, 0^{+} ; \omega}^{\sigma, k,} \mathcal{G} \mathcal{Q}_{2}^{2}(x) \mathcal{J}_{\rho, \lambda, 0^{+} ; \omega}^{\sigma, k} \mathcal{G} \mathcal{Q}_{1}^{2}(x) \leq(\gamma \theta+\Upsilon \Theta)^{2}\left(\mathcal{J}_{\rho, \lambda, 0^{+} ; \omega}^{\sigma, k, \mathcal{G}} \mathcal{G} \mathcal{Q}_{2} \mathcal{Q}_{1}(x)\right)^{2},
$$

which implies the second inequality of (3.18). 
Part (b) follows from the assumption that

$$
\mathcal{G}(t) \mathcal{G}(\eta)\left(\Upsilon \mathcal{Q}_{2}(t)-\theta \mathcal{Q}_{1}(\eta)\right)\left(\Theta \mathcal{Q}_{1}(\eta)-\gamma \mathcal{Q}_{2}(t)\right) \geq 0 \quad \text { for all } t, \eta>0,
$$

which implies that

$$
\begin{aligned}
& \gamma \Upsilon \mathcal{G}(t) \mathcal{G}(\eta) \mathcal{Q}_{2}^{2}(t)+\theta \Theta \mathcal{G}(t) \mathcal{G}(\eta) \mathcal{Q}_{1}^{2}(\eta) \\
& \quad \leq \gamma \theta \mathcal{G}(t) \mathcal{G}(\eta) \mathcal{Q}_{2}(t) \mathcal{Q}_{1}(\eta)+\Upsilon \Theta \mathcal{G}(t) \mathcal{G}(\eta) \mathcal{Q}_{2}(t) \mathcal{Q}_{1}(\eta) .
\end{aligned}
$$

Multiplying both sides of (3.28) by

$$
\frac{\vartheta^{\prime}(t) \vartheta^{\prime}(\eta)}{(\vartheta(x)-\vartheta(t))^{1-\frac{\lambda}{k}}(\vartheta(x)-\vartheta(\eta))^{1-\frac{\delta}{k}}} \mathcal{F}_{\rho, \lambda}^{\sigma, k}\left[\omega(\vartheta(x)-\vartheta(\eta))^{\rho}\right] \mathcal{F}_{\rho, \delta}^{\sigma, k}\left[\omega(\vartheta(x)-\vartheta(\eta))^{\rho}\right]
$$

and then integrating the obtained inequality with respect to $t$ and $\eta$ over $(0, x)$ give the desired inequality (3.20).

For parts (c) and (d), it follows from the Cauchy inequality that

$$
\begin{aligned}
& \left(\mathcal{J}_{\rho, \lambda, 0^{+} ; \omega}^{\sigma, k, \vartheta} \mathcal{G} \mathcal{Q}_{1}(x)\right)^{2} \leq \mathcal{J}_{\rho, \lambda, 0^{+} ; \omega}^{\sigma, k, \vartheta} \mathcal{G}(x) \mathcal{J}_{\rho, \lambda, 0^{+} ; \omega}^{\sigma, k, \vartheta} \mathcal{G} \mathcal{Q}_{1}^{2}(x), \\
& \left(\mathcal{J}_{\rho, \lambda, 0^{+} ; \omega}^{\sigma, k, \vartheta} \mathcal{G} \mathcal{Q}_{2}(x)\right)^{2} \leq \mathcal{J}_{\rho, \lambda, 0^{+} ; \omega}^{\sigma, k, \vartheta} \mathcal{G}(x) \mathcal{J}_{\rho, \lambda, 0^{+} ; \omega}^{\sigma, k, \vartheta} \mathcal{G} \mathcal{Q}_{2}^{2}(x) .
\end{aligned}
$$

From parts (a) and (b), together with the preceding two inequalities, we get

$$
\begin{aligned}
\gamma & \Upsilon\left(\mathcal{J}_{\rho, \lambda, 0^{+} ; \omega}^{\sigma, k, \mathcal{G}} \mathcal{G} \mathcal{Q}_{2}(x)\right)^{2}+\theta \Theta\left(\mathcal{J}_{\rho, \lambda, 0^{+} ; \omega}^{\sigma, k, \vartheta} \mathcal{G} \mathcal{Q}_{1}(x)\right)^{2} \\
& \leq \gamma \Upsilon \mathcal{J}_{\rho, \lambda, 0^{+} ; \omega}^{\sigma, k, \vartheta} \mathcal{G}(x) \mathcal{J}_{\rho, \lambda, 0^{+} ; \omega}^{\sigma, k, \vartheta} \mathcal{G} \mathcal{Q}_{1}^{2}(x)+\theta \Theta \mathcal{J}_{\rho, \lambda, 0^{+} ; \omega}^{\sigma, k, \vartheta} \mathcal{G}(x) \mathcal{J}_{\rho, \lambda, 0^{+} ; \omega}^{\sigma, k,} \mathcal{G} \mathcal{Q}_{2}^{2}(x) \\
& \leq(\gamma \theta+\Upsilon \Theta) \mathcal{J}_{\rho, \lambda, 0^{+} ; \omega}^{\sigma, k, \vartheta} \mathcal{G}(x) \mathcal{J}_{\rho, \lambda, 0^{+} ; \omega}^{\sigma, k, \vartheta} \mathcal{G} \mathcal{Q}_{2} \mathcal{Q}_{1}(x),
\end{aligned}
$$

which implies (3.20). Furthermore, we have

$$
\begin{aligned}
\gamma & \Upsilon\left(\mathcal{J}_{\rho, \lambda, 0^{+} ; \omega}^{\sigma, k, \mathcal{G}} \mathcal{Q}_{2}(x)\right)^{2}+\theta \Theta\left(\mathcal{J}_{\rho, \lambda, 0^{+} ; \omega}^{\sigma, k, \vartheta} \mathcal{G} \mathcal{Q}_{1}(x)\right)^{2} \\
& \leq \gamma \Upsilon \mathcal{J}_{\rho, \lambda, 0^{+} ; \omega}^{\sigma, k, \vartheta} \mathcal{G}(x) \mathcal{J}_{\rho, \lambda, 0^{+} ; \omega}^{\sigma, k, \vartheta} \mathcal{G} \mathcal{Q}_{1}^{2}(x)+\theta \Theta \mathcal{J}_{\rho, \lambda, 0^{+} ; \omega}^{\sigma, k, \vartheta} \mathcal{G}(x) \mathcal{J}_{\rho, \lambda, 0^{+} ; \omega}^{\sigma, k, \vartheta} \mathcal{G} \mathcal{Q}_{2}^{2}(x) \\
& \leq(\gamma \theta+\Upsilon \Theta) \mathcal{J}_{\rho, \lambda, 0^{+} ; \omega}^{\sigma, k, \vartheta} \mathcal{G} \mathcal{Q}_{1}(x) \mathcal{J}_{\rho, \lambda, 0^{+} ; \omega}^{\sigma, k, \vartheta} \mathcal{G} \mathcal{Q}_{2}(x),
\end{aligned}
$$

which implies (3.21).

\section{Concluding remarks}

This section is dedicated to several particular cases of the main consequences derived in Sects. 2 and 3.

I. If we choose $\omega=0, \lambda=\alpha$, and $\sigma(0)=1$, then under the assumptions of Theorem 2.4, we get the result for one-sided generalized $k$-fractional integral proposed by Rashid et al. [17].

II. If we choose $\omega=0, \lambda=\alpha, \sigma(0)=1$, and $k=1$, then under the assumptions of Theorem 2.4, we get the result for one-sided generalized Riemann-Liouville fractional integral proposed by Kacar et al. [31]. 
III. If we choose $\omega=0, \lambda=\alpha, \sigma(0)=1$, and $\vartheta(t)=\frac{t^{s+1}}{s+1}(s \in \mathbb{R} \backslash\{-1\})$, then under the assumptions of Theorem 2.4, we get the result for one-sided generalized $(k, s)$-fractional integral proposed by Mubeen and Iqbal [32].

$I V$. If we choose $\omega=0, \lambda=\alpha, \sigma(0)=1, \vartheta(t)=\frac{t^{s+1}}{s+1}(s \in \mathbb{R} \backslash\{-1\})$, and $k=1$, then under the assumptions of Theorem 2.4, we get the result for one-sided Katugampola fractional integral proposed by Dubey and Goswami [33].

$V$. If we choose $\omega=0, \lambda=\alpha, \sigma(0)=1, \vartheta(t)=t$, and $k=1$, then under the assumptions of Theorem 2.4, we get the result for one-sided Riemann-Liouville fractional integral proposed by Tariboon et al. [34].

More related results can be derived by using similar methods in Sects. 2 and 3, and we leave the details to the interested readers.

\section{Conclusion}

In the paper, we established new Grüss-type fractional integral inequalities and several other associated variants by employing the generalized fractional integral functions having the Raina function in its kernel. Furthermore, we derived numerous novel variants for the monotonicity of functions. Numerous particular cases can be discussed with consideration of Remarks 1.6 and 1.7, which we can supposed as a significant modification of the earlier consequences. For an appropriate choice of $\omega, \lambda$, and $\sigma(0)=1$, we can acquire several novelties, which need further investigations. We hope that novelties concerned with our generalizations can bring revolutionary development and also be implemented in differential and difference equations.

\section{Acknowledgements}

The authors would like to express their sincere thanks to the editor and anonymous reviewers for their helpful comments and suggestions.

Funding

The work was supported by the Natural Science Foundation of China (Grant Nos. 11401192, 61673169).

Availability of data and materials

Not applicable.

Competing interests

The authors declare that they have no competing interests.

Authors' contributions

All authors contributed equally to the writing of this paper. All authors read and approved the final manuscript.

Author details

${ }^{1}$ School of Science, Hunan City University, 413000 Yiyang, China. ${ }^{2}$ Department of Mathematics, Government College University, Faisalabad, Pakistan. ${ }^{3}$ Division of Applied Mathematics, Thu Dau Mot University, Binh Duong Province, Vietnam. ${ }^{4}$ Department of Mathematics, COMSATS University Islamabad, Islamabad, Pakistan. ${ }^{5}$ Department of Mathematics, Lahore College Women University, Jhangh Campus, Lahore, Pakistan. ${ }^{6}$ Department of Mathematics, Huzhou University, Huzhou, China. ${ }^{7}$ Hunan Provincial Key Laboratory of Mathematical Modeling and Analysis in Engineering, Changsha University of Science \& Technology, Changsha, China.

\section{Publisher's Note}

Springer Nature remains neutral with regard to jurisdictional claims in published maps and institutional affiliations.

Received: 16 July 2020 Accepted: 9 November 2020 Published online: 18 November 2020

\section{References}

1. Kumar, S., Atangana, A.: A numerical study of the nonlinear fractional mathematical model of tumor cells in presence of chemotherapeutic treatment. Int. J. Biomath. 13(3), 2050021 (2020). https://doi.org/10.1142/S1793524520500217

2. Ghanbari, B., Atangana, A.: A new application of fractional Atangana-Baleanu derivatives: designing ABC-fractional masks in image processing. Physica A 542, 123516 (2020). https://doi.org/10.1016/j.physa.2019.123516 
3. Atangana, A: Modelling the spread of Covid-19 with new fractal-fractional operators: can the lockdown save mankind before vaccination? Chaos Solitons Fractals 136, Article ID 109860 (2020)

4. Danane, J., Allali, K., Hammouch, Z: Mathematical analysis of a fractional differential model of HBV infection with antibody immune response. Chaos Solitons Fractals 136, Article ID 109787 (2020)

5. Abdeljawad, T., Baleanu, D.: Monotonicity results for fractional difference operators with discrete exponential kernels. Adv. Differ. Equ. 2017, Article ID 78 (2017)

6. Akin, E., Asliyüce, S., Güvenilir, A.F., Kaymakçalan, B.: Discrete Grüss type inequality on fractional calculus. J. Inequal. Appl. 2015, Article ID 174 (2015)

7. Li, X., Mohapatra, R.N., Rodriguez, R.S.: Grüss-type inequalities. J. Math. Anal. Appl. 267, 434-443 (2002)

8. Khan, H., Abdeljawad, T., Tunç, C., Alkhazzan, A., Khan, A.: Minkowski's inequality for the AB-fractional integral operator. J. Inequal. Appl. 2019, Article ID 96 (2019). https://doi.org/10.1186/s13660-019-2045-3

9. Khan, H., Tunç, C., Baleanu, D., Khan, A., Alkhazzan, A.: Inequalities for $n$-class of functions using the Saigo fractional integral operator. Rev. R. Acad. Cienc. Exactas Fís. Nat., Ser. A Mat. 113, 2407-2420 (2019)

10. Grüss, G.: Uber das maximum des absoluten Betrages $\frac{1}{v_{2}-v_{1}} \int_{v_{1}}^{v_{2}} \mathcal{Q}_{1}(z) \mathcal{Q}_{2}(z) d z-\frac{1}{\left(v_{2}-v_{1}\right)^{2}} \int_{v_{1}}^{v_{2}} \mathcal{Q}_{1}(z) d z \int_{v_{1}}^{v_{2}} \mathcal{Q}_{2}(z) d z$ Math. Z. 39, 215-226 (1935)

11. Pachpatte, B.G.: On multidimensional Grüss type inequalities. J. Inequal. Pure Appl. Math. 3, 1-15 (2002)

12. Wang, G., Agarwal, P., Chand, M.: Certain Grüss type inequalities involving the generalized fractional integral operator. J. Inequal. Appl. 2014, Article ID 147 (2014)

13. Vivas-Cortez, M., Kashuri, A., Hernández Hernández, J.E.: Trapezium-type inequalities for Raina's fractional integrals operator using generalized convex functions. Symmetry 12, 1034 (2020). https://doi.org/10.3390/sym12061034

14. Hernández Hernández, J.E., Vivas-Cortez, M.: Hermit-Hadamard inequalities type for Raina's fractional integral operator using $\eta$-convex functions. Rev. Mat. 26(1), 1-19 (2019). https://doi.org/10.15517//rmta.v26i1.35515

15. Baleanu, D., Purohit, S.D., Prajapati, J.C.: Integral inequalities involving generalized Erdelyi-Kober fractional integral operators. Open Math. 14(1), 89-99 (2016)

16. Dahmani, Z:: About some integral inequalities using Riemann-Liouville integrals. Gen. Math. 20(4), 63-69 (2012)

17. Rashid, S., Jarad, F., Noor, M.A., Noor, K.I., Baleanu, D., Liu, J.-B.: Grüss type inequalities for generalized K-fractional integral. Adv. Differ. Equ. 2020, 203 (2020)

18. Rashid, S., Jarad, F., Noor, M.A.: Grüss-type integrals inequalities via generalized proportional fractional operators. Rev. R. Acad. Cienc. Exactas Fís. Nat., Ser. A Mat. 114, 93 (2020). https://doi.org/10.1007/s13398-020-00823-5

19. Rashid, S., Jarad, F., Noor, M.A., Kalsoom, H., Chu, Y.-M.. Inequalities by means of generalized proportional fractional integral operators with respect to another function. Mathematics 7, 1225 (2019). https://doi.org/10.3390/math7121225

20. Butt, S.I., Akdemir, A.O., Bhatti, M.Y., Nadeem, M.: New refinements of Chebyshev-Pólya-Szegö type inequalities via generalized fractional integral operators. J. Inequal. Appl. 2020, 157 (2020)

21. Dokuyucu, M.A.: A fractional order alcoholism model via Caputo-Fabrizio derivative. AIMS Math. 5(2), 781-797 (2019)

22. Raina, R.K.: On generalized Wright's hypergeometric functions and fractional calculus operators. East Asian Math. J. 21(2), 191-203 (2005)

23. Tunç, T., Budak, H., Usta, F., Sarikaya, M.Z.: On new generalized fractional integral operators and related fractiona inequalities. Konuralp J. Math. 8(2), 268-278 (2020)

24. Agarwal, R.P., Luo, M.-J., Raina, R.K.: On Ostrowski type inequalities. Fasc. Math. 204, 5-27 (2016)

25. Kilbas, A.A., Srivastava, H.M., Trujillo, J.J.: Theory and Applications of Fractional Differential Equations. North-Holland Mathematics Studies, vol. 204. Elsevier, Amsterdam (2006)

26. Mubeen, S., Habibullah, G.M.: k-Fractional integrals and applications. Int. J. Contemp. Math. Sci. 7, 89-94 (2012)

27. Sarikaya, M.Z., Dahmani, Z., Kiris, M., Ahmad, F.: (k, s)-Riemann-Liouville fractional integral and applications. Hacet. J. Math. Stat. 45(1), 77-89 (2016)

28. Katugampola, U.N. New approach to a generalized fractional integral. Appl. Math. Comput. 218, 860-865 (2011)

29. Mitrinović, D.S., Pečarić, J.E., Fink, A.M.: Classical and New Inequalities in Analysis. Kluwer Academic, Dordrecht (1993)

30. Dragomir, S.S.: A survey on Cauchy-Bunyakovsky-Schwarz type discrete inequalities. J. Inequal. Pure Appl. Math. 4(3), Article ID 63 (2003)

31. Kacar, E., Kacar, Z., Yildirim, H.: Integral inequalities for Riemann-Liouville fractional integrals of a function with respect to another function. Iran. J. Math. Sci. Inform. 13(1), 1-13 (2018)

32. Mubeen, S., lqbal, S.: Grüss type integral inequalities for generalized Riemann-Liouville $k$-fractional integrals. J. Inequal. Appl. 2016, 109 (2016)

33. Dubey, R.S., Goswami, P.: Some fractional integral inequalities for the Katugampola integral operator. AIMS Math. 4(2) 193-198 (2019). https://doi.org/10.3934/math.2019.2.193

34. Tariboon, J., Ntouyas, S.K., Sudsutad, W.: Some new Riemann-Liouville fractional integral inequalities. Int. J. Math. Math. Sci. 2014, Article ID 869434 (2014) 